\title{
The impact of stellar and AGN feedback on halo-scale baryonic and dark matter accretion in the EAGLE simulations
}

\author{
Ruby J. Wright ${ }^{\star 1,2}$, Claudia del P. Lagos ${ }^{1,2}$, Chris Power ${ }^{1,2}$, Peter D. Mitchell ${ }^{3}$ \\ ${ }^{1}$ International Centre for Radio Astronomy Research (ICRAR), University of Western Australia, Crawley, WA 6009, Australia \\ ${ }^{2}$ ARC Centre of Excellence for All Sky Astrophysics in 3 Dimensions (ASTRO 3D) \\ ${ }^{3}$ Leiden Observatory, Leiden University, P.O. Box 9513, 2300 RA Leiden, the Netherlands
}

Accepted XXX. Received YYY; in original form ZZZ

\begin{abstract}
We use the EAGLE suite of hydrodynamical simulations to analyse accretion rates (and the breakdown of their constituent channels) onto haloes over cosmic time, comparing the behaviour of baryons and dark matter (DM). We also investigate the influence of sub-grid baryon physics on halo-scale inflow, specifically the consequences of modelling radiative cooling, as well as feedback from stars and active galactic nuclei (AGN). We find that variations in halo baryon fractions at fixed mass (particularly their circum-galactic medium gas content) are very well correlated with variations in the baryon fraction of accreting matter, which we show to be heavily suppressed by stellar feedback in low-mass haloes, $M_{\text {halo }} \lesssim 10^{11.5} M_{\odot}$. Breaking down accretion rates into first infall, recycled, transfer and merger components, we show that baryons are much more likely to be smoothly accreted than to have originated from mergers when compared to DM, finding (averaged across halo mass) a merger contribution of $\approx 6 \%$ for baryons, and $\approx 15 \%$ for $\mathrm{DM}$ at $z \approx 0$. We also show that the breakdown of inflow into different channels is strongly dependent on sub-grid physics, particularly the contribution of recycled accretion (accreting matter that has been previously ejected from progenitor haloes). Our findings highlight the dual role that baryonic feedback plays in regulating the evolution of galaxies and haloes: by (i) directly removing gas from haloes, and (ii) suppressing gas inflow to haloes.
\end{abstract}

Key words: galaxies: formation - galaxies: evolution - galaxies: haloes

\section{INTRODUCTION}

\subsection{Motivations \& the current paradigm of mass} assembly

Cosmological simulations focused on the physics of galaxy formation and evolution have proven to be exceptionally powerful predictive tools in extragalactic astrophysics. Hydrodynamical simulations and semi-analytic models are developing in parallel, acting to support observational surveys in constraining the complex baryonic physics that takes place within galaxies, and their consequent observable properties (Somerville \& Davé 2015).

Currently, semi-analytic models (SAMs) are the best suited theoretical tool to investigate the physics behind galaxy formation and evolution on large scales. This is

^ E-mail: ruby.wright@icrar.org largely due to their ability to explore vast regions of parameter space, and produce sizeable galaxy populations without prohibitive computational cost. Many SAMs (e.g. GALFORM: Cole et al. 2000; Lacey et al. 2016, L-GALAXIES: Henriques et al. 2015, and SHARK: Lagos et al. 2018b) build galaxy populations based on dark matter (DM) only simulations, and must accordingly infer the growth of baryons in galaxies based on the DM growth of the host halo. The behaviour of baryons is normally assumed to trace exactly that of DM, with a mass fraction of $f_{\mathrm{b}}=\frac{\Omega_{\mathrm{b}}}{\Omega_{\mathrm{m}}} \approx 0.165$, defined by the parameters of a concordance $\Lambda$ Cold DM $(\Lambda \mathrm{CDM})$ cosmology (Planck Collaboration et al. 2018).

In a no-feedback regime, Crain et al. (2007) show this assumption to be valid in cosmological hydrodynamical simulations. They found that the baryon fraction of haloes within the virial radius is $(\approx 90 \pm 6) \% \times f_{\mathrm{b}}$, independent of halo mass and redshift. This said, the assumption that 
the baryonic mass traces DM has not been extensively explored in modern simulations. The aim of our work is to approach this multi-faceted problem by making use of state-ofthe-art cosmological hydrodynamical simulations (including feedback physics) to investigate gas and DM inflow, with the eventual goal of applying our results to better inform SAMs.

In the standard $\Lambda$ CDM paradigm, the classical picture of matter accumulation on halo-scales is as follows: matter collapses under its own gravity to form overdensities in a filamentary structure referred to as the "cosmic web". In the case of DM, collapse will proceed (typically along the existing filaments) to form virialised, spherical haloes. In the case of baryonic matter, as the gas falls onto these structures, it can either (i) continue to free-fall to the central regions of the halo, like DM (e.g. Katz et al. 2003; Kereš et al. 2005, referred to as "cold-mode" accretion) or (ii) be shock heated at the virial radius to form a semi-stable, pressure supported atmosphere (e.g. Rees \& Ostriker 1977; White \& Rees 1978, referred to as "hot-mode" accretion). Cold-mode accretion is thought to dominate at high redshift, where it is possible for cold, collimated streams to infiltrate hot haloes (e.g. Dekel \& Birnboim 2006; Ocvirk et al. 2008; Kereš et al. 2009; Dekel et al. 2009; van de Voort et al. 2011). Hot-mode accretion is expected in the case where gas infall is supersonic (Binney 1977), which is expected primarily in high halo masses (groups/clusters). For a comprehensive review, see Benson (2010).

Above, we discuss hot- and cold-modes of gas inflow (both "smooth"-mode accretion), but it is also important to consider the mass-growth of haloes and galaxies via mergers or "clumpy" inflow. Hierarchical mass assembly is one of defining processes predicted by a $\Lambda \mathrm{CDM}$ cosmology, with numerical work from Genel et al. (2010) using the Millennium and Millennium-II simulations suggesting that $60 \%$ of total halo mass growth is the result of mergers (in a DM-only regime). With an implementation of baryonic physics, van de Voort et al. (2011) show that gas accretion onto haloes is predominantly "smooth-mode", with merger-driven baryon growth (with mass ratios above $1: 10$ ) being only significant in groups and clusters.

In order to model gas inflow onto haloes and galaxies, it is also important to consider gas outflows. When stellar and active galactic nuclei (AGN) feedback occurs in galaxies, the energetic outflows can produce "fountains" of matter which will are eventually "recycled" into the galaxy (e.g. Rubin et al. 2012; Emonts et al. 2015; Pereira-Santaella et al. 2016; Anglés-Alcázar et al. 2017). The balance between gas inflow, outflow and recycling define the "baryon cycle" - the time evolution and breakdown of baryons (and their properties) in different reservoirs, at both galaxy- and halo-scales. The baryon cycle eventually sets the scaling between the stellar mass, gas mass and halo mass of galaxies, with recent simulation-based work highlighting the need for a more complete understanding of the interaction between inflows and outflows in the circum-galactic medium (CGM, see Mitchell et al. 2020b and Mitchell et al. 2020a).

\subsection{Quantifying baryon accretion: theoretical and observational literature}

Continuity arguments form the basis of many phenomenological efforts to model galaxy evolution in a cosmological context (Bouché et al. 2010; Davé et al. 2012; Lilly et al. 2013; Peng \& Maiolino 2014). Analytical approaches typically involve a halo inflow term, and the eventual accretion onto the central galaxy is counteracted by preventative feedback terms. As an illustration, Davé et al. (2012) adopt the following prescription:

$\dot{M}_{\text {in }}=\dot{M}_{\text {grav }}-\dot{M}_{\text {prev }}+\dot{M}_{\text {recyc }}$,

where $\dot{M}_{\text {in }}$ is the net inflow rate to the galaxy, $\dot{M}_{\text {grav }}$ is the total mass inflow rate onto the host halo (see Equation 2), $\dot{M}_{\text {prev }}$ represents the growth rate of intra-halo/CGM gas (gas inside the halo, but outside the central galaxy), and $\dot{M}_{\text {recyc }}$ is the rate at which previously ejected gas (now part of the CGM) is reincorporated into the central galaxy's interstellar medium (ISM). The preventative feedback parameter, $\zeta$, is then defined to be $\zeta \equiv 1-\frac{\dot{M}_{\text {prev }}}{M_{\text {grav }}}$. The $\dot{M}_{\text {grav }}$ term is derived from Extended Press Schechter (EPS) theory (Neistein et al. 2006), taking into account the assumed cosmology and predicted clustering of matter into spherical haloes. It is described by Equation 2 from Dekel et al. (2009):

$\frac{\dot{M}_{\text {grav }}}{M_{\text {halo }}}=0.47 f_{\mathrm{b}}\left(\frac{M_{\text {halo }}}{10^{12} M_{\odot}}\right)^{0.15}\left(\frac{1+z}{3}\right)^{2.25} \mathrm{Gyr}^{-1}$.

This prescription highlights the following points: (i) the efficiency of gas inflow $\left(\dot{M}_{\text {grav }} / M_{\text {halo }}\right)$ onto haloes increases modestly with halo mass (with a power-law index $=0.15$ ); (ii) the efficiency of gas inflow onto haloes decreases over cosmic time, and (iii) the amount of gas that enters a galaxy's ISM is modulated by preventative feedback from the galaxy. The latter can be broken down into: photoionisation, stellar feedback (winds \& heating), AGN feedback (winds \& heating) and thermal pressure from gravitational compression. More recently, some empirical models have begun to allow a connection between galaxy star formation rates (SFRs) and halo-scale inflow rates (Moster et al. 2018; Behroozi et al. 2019).

Several simulation-based studies have investigated the influence of feedback physics on gas accretion. It is important to differentiate between gas accretion onto haloes and gas accretion onto galaxies (effectively, the $\dot{M}_{\text {grav }}$ term and $\dot{M}_{\text {in }}$ term, respectively). The interplay between feedback mechanisms and gas inflow within the halo environment is explored in depth in van de Voort et al. (2011), who focus on central halo- and galaxy-scale mass inflow (gross mass flux of gas particles into these structures over a discrete timestep). They define haloes as per the SUBFIND algorithm (Springel et al. 2001; Dolag et al. 2009), and the ISM as gas that is dense enough (most of which will be star forming) within $0.15 R_{200}$. With a range of GADGET-based (Springel 2005) hydrodynamical simulations implementing varying physics, they find: (i) that the efficiency of gas accretion onto haloes is only weakly dependent on halo mass (and at halo masses above $10^{11} M_{\text {halo }}$, is fairly insensitive to feedback), and (ii) that accretion rates to galaxies are much more sensitive to somewhat uncertain feedback/cooling mechanisms.

Nelson et al. (2015) use two simulations based on the moving mesh AREPO code, one with both stellar \& AGN feedback (similar to the ILLUSTRIS simulation), and one with neither stellar nor AGN feedback. They focus on inflows at galaxy-scales for centrals, and measure net accretion rate using a tracer-particle method, counting the number of in- 
ward crossings of a boundary at $r=0.15 r_{\mathrm{vir}}$, subtract the number of outgoing crossings. They find that the fraction of mass delivered via smooth accretion is consistently lower in the presence of feedback by a factor of 2 at all redshifts, and that at $z<1$, cold-mode accretion in the feedback run is reduced to almost zero. At the halo-scale, they show for haloes of mass $10^{11.3} M_{\odot}<M_{\text {halo }}<10^{11.4} M_{\odot}$ that inflow rates to the virial sphere are similar between their feedback and nofeedback runs at early times, but diverge by $\approx 0.25$ dex at $z \approx 0$, where inflow rates are suppressed in the presence of feedback.

More recently, Correa et al. (2018) investigate gas accretion onto haloes and central galaxies in the EAGLE suite of hydrodynamical simulations (Schaye et al. 2015; Crain et al. 2015). To calculate accretion rates to each halo, they find the flux of particles from outside the Friends-Of-Friends (FOF) halo to within the virial sphere, $R_{200}$, crit . With this methodology, they find that gas accretion at the halo-scale in the reference physics run deviates from a DM-only prediction (Correa et al. 2015a,b,c), with inflow suppressed for lower mass haloes. Interestingly, they show at the galaxy-scale for $z<2$ for haloes with $10^{11.7} M_{\odot}<M_{\text {halo }}<10^{12.7} M_{\odot}$ that the accretion rate to galaxies remains roughly independent of halo mass, while for haloes of mass $M_{\text {halo }}<10^{11.7} M_{\odot}$ or $M_{\text {halo }}>10^{12.7} M_{\odot}$, there is an obvious positive scaling of accretion rate with halo mass. They attribute the flattening at the galaxy-scale to AGN feedback, as this flattening vanishes in the no-AGN variant. They do not focus on comparing accretion rates onto haloes between different runs with varying feedback physics.

Focusing instead on satellite galaxies, gas accretion and its environmental dependence at the galaxy-scale was investigated in van de Voort et al. (2017) using the reference EAGLE simulation (Schaye et al. 2015; Crain et al. 2015). Using adjacent snapshots, they define gas accretion onto the galaxy to be the number of newly star-forming ISM particles found in a satellite, which were not star-forming at the previous snapshot. They found galaxies that become satellites in massive haloes can be "starved" of gas accretion, directly causing their star formation to quench.

Although simulation-based methodologies of measuring inflow are decidedly diverse, it is also clear that the efficiency of inflow scales with the mass of a halo in a non-linear manner, eventually acting to set the relationship between stellar and halo mass. Most simulation-based work has indicated that feedback has little impact on the accretion rates at the halo-scale, unlike the galaxy-scale. The reader should note that the technique of calculating inflow, binning, and averaging can have significant impact on the quantitative measurements of accretion rates, and that some quantitative tension between works could plausibly be explained by the specific methodology used.

Observational constraints on gas accretion onto haloes and galaxies are limited. Direct measurement of inflow is exceedingly difficult given the typically small covering fraction of accreting material, the predominance of outflows, and the necessity for individual high signal-to-noise $(\mathrm{S} / \mathrm{N})$ spectra (Rubin et al. 2012). Additionally, accreting matter typically has a relatively weak kinematic signature compared to the galaxy's systematic redshift, unlike (typically high-velocity) feedback-driven outflows. Observational studies have confirmed the occurrence of inflows around the Milky Way (e.g.
Lehner \& Howk 2011; Lockman et al. 2008). Others have investigated extra-galactic sources with various methodologies, for instance direct "down-the-barrel" measurements of redshifted absorption lines (e.g. Rubin et al. 2012, for a review see Rubin 2017), and quasar-galaxy pairs (e.g. Bouché et al. 2013, 2016; Ho et al. 2017; Rahmani et al. 2018; Zabl et al. 2019). At this stage, studies of this nature are difficult to discuss quantitatively, with some work focusing on individual galaxies, and others limited to a sample of tens of galaxies (Faucher-Giguère 2017). Despite the scarcity of direct observations, it is clear from the star formation histories and rates of galaxies that accretion is expected to be a major regulator of galaxy growth (Lilly et al. 2013), and that accretion and outflows are likely interacting in the CGM (e.g. Martin et al. 2019; Kacprzak et al. 2019; Pointon et al. 2019; Nielsen et al. 2020).

The content we present in this paper is structured as follows. In $\S 2$, we introduce (i) the EAGLE hydrodynamical simulation suite and the sub-grid models that are relevant to this study, (ii) VELOCIRAPTOR and TrEeFrog: the phasespace structure finder we use to identify bound haloes and substructures (and its accompanying halo merger tree generator), and (iii) CHUMm: the code we use to calculate and analyse accretion rates onto haloes in EAGLE. $\S 3$ compares the build-up of gas and DM in EAGLE runs with fiducial sub-grid physics. $\S 4$ looks at the impact of including various physical processes in the hydrodynamical simulation when compared to the results of $\S 3$. In $\S 5$, we discuss the implications of our findings on semi-analytic prescriptions of baryon build-up in galaxies. In $\S 6$, we conclude what these findings tell us about the growth of baryonic matter in galaxies, and future direction. Lastly, in Appendices A, B, C and D, we explore the sensitivity of our measurements to (i) number statistics, (ii) simulation spatial/mass resolution, (iii) simulation temporal resolution, and (iv) measurement methodology respectively.

\section{METHODS}

\subsection{The EAGLE simulations}

The EAGLE (Evolution and Assembly of GaLaxies and their Environments) simulation suite (Schaye et al. 2015; Crain et al. 2015) is a collection of cosmological hydrodynamical simulations that follow the evolution of galaxies and cosmological structure down to $z=0$. The ANARCHY (Schaller et al. 2015) set of revisions (designed to correct for "classical" SPH issues) were implemented on the GADGET3 tree-SPH code (Springel 2005) to perform the EAGLE simulations over a variety of periodic volumes and resolutions. EAGLE adopts the parameters of a $\Lambda$ CDM universe from Planck Collaboration et al. (2014), with initial conditions outlined in Jenkins (2013). Sub-grid physics modules were implemented to treat processes that are important for galaxy formation and evolution, but occur on scales below the resolution-scale of the simulation. These include: (i) radiative cooling and photoheating, (ii) star formation, (iii) stellar evolution and enrichment, (iv) stellar feedback, and (v) supermassive black hole (SMBH) growth and AGN feedback. Below, we provide a brief description of how these mechanisms are modelled in EAGLE. 


\begin{tabular}{|c|c|c|c|c|c|c|c|c|c|c|c|}
\hline Run Name & $\begin{array}{c}L_{\mathrm{box}} \\
{[\mathrm{cMPc}]}\end{array}$ & $N_{\text {part }}$ & $\begin{array}{l}m_{\mathrm{DM}} \\
{\left[\boldsymbol{M}_{\odot}\right]}\end{array}$ & $\begin{array}{l}m_{\text {gas }} \\
{\left[M_{\odot}\right]}\end{array}$ & $\begin{array}{c}\epsilon \\
{[\mathrm{pkpc}]}\end{array}$ & $\begin{array}{c}f_{\text {th }} \\
(\min )\end{array}$ & $\begin{array}{c}f_{\mathrm{th}} \\
(\max )\end{array}$ & $\begin{array}{c}\Delta T_{\mathrm{SN}} \\
{[\mathrm{K}]}\end{array}$ & $\begin{array}{c}\Delta T_{\mathrm{AGN}} \\
{[\mathrm{K}]}\end{array}$ & $\mathrm{RC}$ & $\begin{array}{l}N_{\text {field halo }}(z=0) \\
\left(M_{\text {halo }}>10^{9} M_{\odot}\right)\end{array}$ \\
\hline L25-REF & 25 & $376^{3}$ & $9.7 \times 10^{6}$ & $1.8 \times 10^{6}$ & 0.7 & 0.3 & 3 & $10^{7.5}$ & $10^{8.5}$ & $\checkmark$ & 9,807 \\
\hline L50-REF & 50 & $752^{3}$ & $9.7 \times 10^{6}$ & $1.8 \times 10^{6}$ & 0.7 & 0.3 & 3 & $10^{7.5}$ & $10^{8.5}$ & $\checkmark$ & 74,743 \\
\hline L50-NOAGN & 50 & $752^{3}$ & $9.7 \times 10^{6}$ & $1.8 \times 10^{6}$ & 0.7 & 0.3 & 3 & $10^{7.5}$ & $\mathrm{~N} / \mathrm{A}$ & $\checkmark$ & 74,351 \\
\hline L50-AGNdT8 & 50 & $752^{3}$ & $9.7 \times 10^{6}$ & $1.8 \times 10^{6}$ & 0.7 & 0.3 & 3 & $10^{7.5}$ & $10^{8}$ & $\bar{\checkmark}$ & 74,788 \\
\hline L50-AGNdT9 & 50 & $752^{3}$ & $9.7 \times 10^{6}$ & $1.8 \times 10^{6}$ & 0.7 & 0.3 & 3 & $10^{7.5}$ & $10^{9}$ & $\bar{\checkmark}$ & 75,645 \\
\hline L25-ONLYAGN & 25 & $376^{3}$ & $9.7 \times 10^{6}$ & $1.8 \times 10^{6}$ & 0.7 & $\mathrm{~N} / \mathrm{A}$ & $\mathrm{N} / \mathrm{A}$ & $\mathrm{N} / \mathrm{A}$ & $10^{8.5}$ & $\checkmark$ & 9,944 \\
\hline L25-WEAKSN & 25 & $376^{3}$ & $9.7 \times 10^{6}$ & $1.8 \times 10^{6}$ & 0.7 & 0.15 & 1.5 & $10^{7.5}$ & $10^{8.5}$ & $\checkmark$ & 9,951 \\
\hline L25-STRONGSN & 25 & $376^{3}$ & $9.7 \times 10^{6}$ & $1.8 \times 10^{6}$ & 0.7 & 0.6 & 6 & $10^{7.5}$ & $10^{8.5}$ & $\checkmark$ & 9,839 \\
\hline L25-NOFB & 25 & $376^{3}$ & $9.7 \times 10^{6}$ & $1.8 \times 10^{6}$ & 0.7 & $\mathrm{~N} / \mathrm{A}$ & $\mathrm{N} / \mathrm{A}$ & $\mathrm{N} / \mathrm{A}$ & $\mathrm{N} / \mathrm{A}$ & $\checkmark$ & 9,362 \\
\hline L25-NONRAD & 25 & $376^{3}$ & $9.7 \times 10^{6}$ & $1.8 \times 10^{6}$ & 0.7 & $\mathrm{~N} / \mathrm{A}$ & $\mathrm{N} / \mathrm{A}$ & $\mathrm{N} / \mathrm{A}$ & $\mathrm{N} / \mathrm{A}$ & $x$ & 10,751 \\
\hline
\end{tabular}

Table 1. Simulation parameters for the EAGLE runs utilised in this paper (Schaye et al. 2015; Crain et al. 2015). $L_{\text {box }}$ is the comoving box size of the simulation; $N_{\text {part }}$ refers to the number of DM particles (and initial number of gas particles); $M_{\mathrm{DM}}$ and $M_{\mathrm{gas}}$ refer to the masses of DM and gas particles in the simulation respectively; $\epsilon$ refers to the Plummer equivalent maximum gravitational smoothing length; $f_{\mathrm{th}}, \min \& f_{\mathrm{th}}$, max are the minimum and maximum supernovae energy transfer fractions (per unit stellar mass); $\Delta T_{\mathrm{SN}} \& \Delta T_{\mathrm{AGN}}$ are the heating temperatures adopted for stellar and AGN feedback; "RC" indicates the inclusion of radiative cooling; and $N_{\text {field halo }}(z=0)$ describes the number of field haloes in each run at the final snapshot.

\begin{tabular}{|c|c|c|c|c|c|c|c|c|c|c|c|}
\hline Run Name & $\begin{array}{c}L_{\mathrm{box}} \\
{[\mathrm{cMPc}]}\end{array}$ & $N_{\text {part }}$ & $\begin{array}{l}m_{\mathrm{DM}} \\
{\left[\boldsymbol{M}_{\odot}\right]}\end{array}$ & $\begin{array}{l}m_{\text {gas }} \\
{\left[M_{\odot}\right]}\end{array}$ & $\begin{array}{c}\epsilon \\
{[\mathrm{pkpc}]}\end{array}$ & $\begin{array}{c}f_{\mathrm{th}} \\
(\mathrm{min})\end{array}$ & $\begin{array}{c}f_{\mathrm{th}} \\
(\max )\end{array}$ & $\begin{array}{c}\Delta T_{\mathrm{SN}} \\
{[\mathrm{K}]} \\
\end{array}$ & $\begin{array}{c}\Delta T_{\mathrm{AGN}} \\
{[\mathrm{K}]} \\
\end{array}$ & $\mathrm{RC}$ & $\begin{array}{l}N_{\text {field halo }}(z=0) \\
\left(M_{\text {halo }}>10^{9} M_{\odot}\right)\end{array}$ \\
\hline L25N752-REF & 25 & $752^{3}$ & $1.2 \times 10^{6}$ & $2.3 \times 10^{5}$ & 0.35 & 0.3 & 3 & $10^{7.5}$ & $10^{8.5}$ & $\checkmark$ & 9,906 \\
\hline L25N752-RECAL & 25 & $752^{3}$ & $1.2 \times 10^{6}$ & $2.3 \times 10^{5}$ & 0.35 & 0.3 & 3 & $10^{7.5}$ & $10^{9}$ & $\checkmark$ & 10,068 \\
\hline (GADGET) L32-NONRAD & 32 & $512^{3}$ & $2.7 \times 10^{7}$ & $5.0 \times 10^{6}$ & 3.0 & $\mathrm{~N} / \mathrm{A}$ & $\mathrm{N} / \mathrm{A}$ & $\mathrm{N} / \mathrm{A}$ & $\mathrm{N} / \mathrm{A}$ & $x$ & 74,724 \\
\hline
\end{tabular}

Table 2. Simulation parameters for the additional convergence and testing runs utilised in this paper, with EAGLE data from Schaye et al. (2015); Crain et al. (2015). $L_{\mathrm{box}}$ is the comoving box size of the simulation; $N_{\text {part }}$ refers to the number of DM particles (and initial number of gas particles); $M_{\mathrm{DM}}$ and $M_{\text {gas }}$ refer to the masses of DM and gas particles in the simulation respectively; $\epsilon$ refers to the Plummer equivalent maximum gravitational smoothing length, $f_{\mathrm{th}}$, $\min \& f_{\mathrm{th}}$, max are the minimum and maximum supernovae energy transfer fractions (per unit stellar mass); $\Delta T_{\mathrm{SN}} \& \Delta T_{\mathrm{AGN}}$ are the heating temperatures adopted for stellar and AGN feedback; "RC" indicates the inclusion of radiative cooling; and $N_{\text {field halo }}(z=0)$ describes the number of field haloes in each run at the final snapshot. Note that the last simulation, L32-NONRAD, is not an EAGLE run, rather a locally run GADGET-based 32 Mpc adiabatic physics box.

Photo-heating and radiative cooling are applied based on the work of Wiersma et al. (2009). This includes the effect of 11 elements in $\mathrm{H}, \mathrm{He}, \mathrm{C}, \mathrm{N}, \mathrm{O}, \mathrm{Ne}, \mathrm{Mg}, \mathrm{Si}, \mathrm{S}, \mathrm{Ca}$, and Fe (Schaye et al. 2015). The effect of radiation from the UV and X-ray background described by Haardt \& Madau (2001) is implemented on each element individually. Since the EAGLE simulations do not provide the resolution to model cold, interstellar gas, a density-dependent temperature floor (normalised to $T=8,000 \mathrm{~K}$ at $n_{\mathrm{H}}=10^{-1} \mathrm{~cm}^{-3}$ ) is imposed. To model star formation, a metallicity-dependent density threshold is set, above which, star formation is locally permitted (Schaye et al. 2015). Gas particles are converted to star particles stochastically, with the star formation rate based on a tuned pressure law (Schaye \& Dalla Vecchia 2008), calibrated to the work of Kennicutt (1998) at $z=0$. The energy feedback from star formation is treated with a thermal energy injection of $10^{51}$ erg per type Ia supernovae (SNIa) event, the amount of which is a function of the IMF adopted (Chabrier 2003). This is implemented in the form of a temperature boost to the surrounding particles of $\Delta T_{\mathrm{SF}}=10^{7.5} \mathrm{~K}$, based on the work of Dalla Vecchia $\&$ Schaye (2012). The number of stars heated is calculated using Equation 3, taken from Equation 8 in Schaye et al. (2015):

$\left\langle N_{\text {heat }}\right\rangle \approx 1.3 f_{\text {th }}\left(\frac{\Delta T}{10^{7.5} \mathrm{~K}}\right)^{-1}$, where $f_{\text {th }}$ is the fraction of the total amount of energy from core collapse supernovae per unit stellar mass that is injected on average. $f_{\text {th }}$ varies between set minimum and maximum values (see Tables 1 and 2), the value in this range calculated based on local ISM properties.

SMBHs are seeded in EAGLE when a DM halo exceeds a virial mass of $10^{10} \mathrm{~h}^{-1} \mathrm{M}_{\odot}$, with the seed SMBHs having an initial mass of $10^{5} \mathrm{~h}^{-1} \mathrm{M}_{\odot}$. Subsequently, SMBHs can grow via Eddington-limited-accretion (Schaye et al. 2015), as well as mergers with other SMBHs, according to work by Springel et al. (2005). Similar to stellar feedback, AGN feedback in EAGLE also involves the injection of thermal energy into particles surrounding the SMBH in the form of temperature boost of $\Delta T_{\mathrm{BH}}=10^{8.5} \mathrm{~K}$ (in the reference physics run; Schaye et al. 2015). The rate of energy injection (which directly sets the number of heated particles) from AGN is determined using the SMBH accretion rate, and a fixed energy conversion efficiency, as in Equation 4:

$\frac{\Delta E}{\Delta t}=\epsilon_{\mathrm{f}} \epsilon_{\mathrm{r}} \dot{m}_{\mathrm{accr}} c^{2}$

where $\dot{m}_{\text {accr }}$ is a modified Bondi-Hoyle accretion rate (see Equations 9,10 in Schaye et al. 2015), and $\epsilon_{\mathrm{f}} \epsilon_{\mathrm{r}}=0.015$.

There are several free parameters in a number of the EAGLE sub-grid modules, and these are calibrated (separately for the standard resolution runs and the L25N752RECAL run) to match $z \approx 0$ observations of (i) the galaxy 
stellar mass function, (ii) the galaxy size-mass relation, and (iii) the galaxy $\mathrm{BH}$ mass - stellar mass relation. We make use of 10 standard resolution EAGLE runs, all with varying feedback physics (see Table 1) but identical starting mass resolution, and a number of supplementary runs with varying mass and temporal resolution (see Table 2). For the remainder of the paper, we refer to each run by their identifier in the "run-name" columns for brevity. We include the mass distributions of field haloes in 4 of the standard and supplementary runs in Appendix A - specifically those of L50-REF, L25-REF, L32-NONRAD, and L25N752-RECAL. Mass distributions for all standard resolution L50 (L25) runs are comparable to the L50 (L25)-REF distribution. We check the convergence of our results with the higher resolution EAGLE variants, with $752^{3}$ particles in a $25 \mathrm{Mpc}$ box in Appendix B. We also use a non-radiative GADGET-based $32 \mathrm{Mpc}$ box (with $512^{3}$ particles) to check temporal convergence (Appendix C), and for enhanced number statistics compared to the EAGLE L25-NONRAD box in $\S 4$.

\subsection{Structure finding \& halo trees with VELOCIRAPTOR and TrEeFrog}

We identify haloes and subhaloes in the EAGLE runs using VELOCIRAPTOR (Elahi et al. 2011, 2019a), a 6D friends of friends (6D-FOF) structure finding algorithm (that is to say, we do not make use of halo and subhalo information provided in the EAGLE public catalogues, outlined in McAlpine et al. 2016). VELOCIRAPTOR first uses a 3DFOF algorithm (Davis et al. 1985) to identify field haloes. Two particles $i$ and $j$ are "linked" together if they satisfy the condition:

$\frac{\left(\mathbf{x}_{i}-\mathbf{x}_{j}\right)^{2}}{l_{x}^{2}}<1 \quad$ (where $l_{x}=0.2 \times$ inter - particle spacing $)$.

3D-FOF algorithms can spuriously link dynamically distinct objects together through tenuous particle bridges. Thus, for each 3D-FOF object, VELOCIRAPTOR subsequently applies a $6 \mathrm{D}-\mathrm{FOF}$ algorithm (including spatial and velocity information) in order to separate virialised structures (Elahi et al. 2019a). The velocity dispersion is calculated for each 3DFOF object $k, \sigma_{v, k}$, and particles within the 6D-FOF are linked together if:

$\frac{\left(\mathbf{x}_{i}-\mathbf{x}_{j}\right)^{2}}{l_{x}^{2}}+\frac{\left(\mathbf{v}_{i}-\mathbf{v}_{j}\right)^{2}}{l_{v}^{2}}<1 \quad\left(\right.$ where $l_{v}=\alpha_{v} \sigma_{v, k}$ and $\left.\alpha_{v} \approx 1.25\right)$.

An unprocessed 3D-FOF halo will typically contain numerous density peaks, some of which may reside outside the virial radius centred on the inner-most minimum potential. Once the 6D-FOF algorithm has been run over a 3D-FOF object, each of the nested density peaks will be identified as "sub-haloes" of the parent halo (with the notable exception of the central 6D-FOF object, which remains identified as the parent halo). For our work, we make use of the fact that VELOCIRAPTOR can return the IDs of particles which constitute a $6 \mathrm{D}-\mathrm{FOF}$ object. To link haloes through time, we use the halo merger tree code TreeFrog (Elahi et al. 2019b), developed to work on the outputs of VELOCIRAPTOR. This code compares the particles in haloes across multiple snapshots by calculating a "merit" based on the fraction of par- ticles that are shared by two (sub)haloes $i$ and $j$ at different times. In circumstances where several matches are identified for one (sub)halo with similar merits (e.g. mergers of several similar mass haloes), TREEFrog ranks particles based on their binding energy. This combined information is then used to estimate a merit function, which makes use of the total number of particles shared, and their binding energy information (see Eq. 3 in Elahi et al. 2019b).

\subsection{Accretion calculations with $\mathrm{CHUMM}$}

In order to calculate accretion rates onto haloes in our simulations, we developed and used the code package CHUMM (Code for Halo AccUMulation of Mass, available at https: //github.com/RJWright25/CHUMM). We focus primarily on the build-up of matter on halo-scales. Methodologies to calculate inflow rates to structure in cosmological simulations can be categorised as either (i) Eulerian, or (ii) Lagrangian in nature. The Eulerian method involves an instantaneous calculation of inflowing mass flux at a defined boundary, while the Lagrangian approach involves a calculation of inflowing mass flux, over a discrete time interval. Our method for calculating accretion flux is Lagrangian in nature, with a number of (optional) additional conditions imposed on inflow particles. As discussed by Mitchell et al. (2020b) in the context of outflows, the Lagrangian method yields more accurate measurement of time-integrated flux, and is less sensitive to the stochasticity of instantaneous particle behaviour at the boundary of haloes. With Lagrangian approaches, it is important to consider the time interval used - van de Voort et al. (2011) use intervals of up to $\approx 1.5 \mathrm{Gyr}$, while van de Voort et al. (2017) and Correa et al. (2018) use a varying $\Delta t$, corresponding to adjacent EAGLE snapshots. We also elect to calculate accretion over the interval between adjacent EAGLE snapshots (29 snapshots from $z=20$ to $z=0$ ), corresponding to a $\Delta t$ ranging between $\approx 250 \mathrm{Myr}$ at minimum (at $z \approx 4$ ), and $\approx 0.9 \mathrm{Gyr}$ at maximum $($ at $z \approx 0$ ). The varying timesteps in EAGLE that we use to calculate accretion rates correspond to $0.5-1.0$ times a halo dynamical time across the full redshift range that we analyse, and thus, for the purposes of our work, we don't believe there is a need for higher cadence intervals. To investigate the sensitivity of our accretion calculations to the time interval, we used a higher cadence (200 snapshot) non-radiative simulation and looked at temporal convergence in Appendix C. We find that while the normalisation of accretion rates can change slightly with the interval, our results are not sensitive to the choice. Additionally, we refer the reader to the work of Mitchell et al. (2020a), who analyse inflow with higher cadence full-physics EAGLE runs and find similar temporal convergence properties to our L32-NONRAD run.

To identify haloes, CHUMm either uses the outputs from VELOCIRAPTOR and selects particle members of 6D-FOF objects, or CHUMM can use a spherical overdensity (SO) criterion, where particles within a spherical region defined by $R_{\text {vir }}$ are selected as constituting a halo. By default, VELOCIRAPTOR returns particle lists for field haloes, with substructure particles removed or "deblended". For field haloes with substructure, we define the full FOF (with substructure) by re-introducing substructure particles to the host. For the SO criterion, we use the VELOCIRAPTOR halo catalogue to iterate through each halo, $i$, determine its cen- 
tral position $\mathbf{x}_{i}$ and virial radius, $r_{200, i}$, and then search for the particles $j$ (with position $\mathbf{x}_{j}$ ) that satisfy the condition $\left(\frac{\left|\mathbf{x}_{i}-\mathbf{x}_{j}\right|}{r_{200, i}}\right)<f$, where CHUMm can take a list of user defined $f$ values. Particles satisfying this condition then constitute the SO definition of the halo $i$. We compare results using different halo definitions (i.e. 6D-FOF particles or spherical overdensity regions with boundaries at fractions of $\left.R_{200}\right)$ in Appendix D, and find results to change slightly (but not qualitatively) between methods. We elect to proceed with the FOF-based boundary for accretion, as we believe this more consistently reflects the nature of accretion over different halo mass scales.

The algorithm CHUMm uses to calculate accretion rates is summarised in Figure 1. Recall each simulation has a finite number of snapshots $N$ (hereafter snaps) that encode the physical state of the system at times $t_{0}<t<t_{N}$, and we equate snap $n$ with the state of the system at lookback time $t=t_{n}$. To calculate accretion rates onto haloes at snap $n$, we identify accretion candidates as the particles that exist in the halo at snap $n$ as per the definition above, but did not exist in the halo at snap $n-d$ ( $d$ being the snap "depth" of the calculation, i.e. the difference in snap index, chosen as 1 unless otherwise stated). The summed mass of these candidate particles, normalised by $\Delta t=t_{n-d}-t_{n}$ (where $t_{n}$ represents the lookback time at snap $n$ ), constitutes the raw gross total accretion rate of the halo at snap $n$. Accretion rates are split by particle type, with the particle type categorised at the initial snap $n-d$, before undergoing any processing in the halo (such that gas particles at snap $n-d$ which were transformed to star particles by snap $n$ would be considered gas inflow, not stellar inflow). We experimented with imposing a number of radial velocity cuts on accretion candidates at the initial snap $n-d$ (to ensure particles are travelling towards the halo's center-of-mass at a sufficient rate): $v_{\text {rad, in, } \mathrm{n}-\mathrm{d}}>f \times v_{\max }$, halo. We found that for a range of reasonable $f$ fractions of the maximum halo circular velocity $v_{\text {max, halo }}$, our inflow results were not significantly impacted. We also experimented with requiring accretion candidates to remain in the halo they were considered accreted to at the subsequent snapshot (i.e. to remain in the halo for an additional $t_{n}-t_{n+1}$ to be considered as "stable" accretion), and again saw only a slight change in the normalisation of accretion rates, rather than any qualitative alterations to trends. When testing this requirement, the effect was quantitatively the largest $(\approx 0.1 \mathrm{dex})$ on the non-radiative boxes, where stochastic flux of particles at the halo boundary (both inwards and outwards) are more common-place in the absence of radiative cooling. Even in the non-radiative runs, however, we saw no qualitative change in the results. As such, we elected not impose the velocity or stability criteria for our results (unless otherwise stated), in order to make our results minimally complex to interpret. Finally, for the FOF-based calculation, we are able to categorise the nature of the inflow particles (their accretion "channel") based on (i) their host at snap $n-d$, and (ii) their processing history. The particle's host at snap $n-d$ determines the origin of accretion as either from the field, or from another VELOCIRAPTOR structure (the particles of each origin we refer to as "cosmological"/"smooth" or "merger"/"clumpy" accretion particles respectively).

For the "cosmological"/"smooth" accretion case, a par- ticle is considered "processed" if it has existed in any halo (as defined by VELOCIRAPTOR) up to and including snap $n-d$ (the initial snap), and "unprocessed"/first infall" otherwise. Commonly the term "pristine" is used to describe the accretion channel of metal-poor, un-enriched particles, however we elect to use the term "first infall" for our "unprocessed" channel to recognise that these particles are only "unprocessed" insofar as VELOCIRAPTOR's ability to identify bound structures, ultimately limited by the finite mass resolution of the simulation. We show the relevant peak in the halo mass functions from each run from VELOCIRAPTOR in Appendix A, corresponding to $M_{\text {halo }} \approx 10^{8.5} M_{\odot}$ at $z \approx 0$ in the standard resolution EAGLE variants. We can further decompose the "processed" channel of cosmological accretion into a "recycled" and "transfer" component - for particles which were previously processed in a progenitor (main, non-main, or a satellite) halo ("recycled" accretion) and those that were previously processed in an unrelated halo ("transferred" accretion) respectively. In a future paper, we explore the physical properties of accreting particles as a function of inflow channels, and find that processed particles (according to our definition) are clearly metal-enriched relative to the first-infall particles, giving us confidence that our methodology is physically-motivated.

While our definitions of accretion channels are similar to those in (Mitchell et al. 2020a), we remark that the definition of "transfer" in previous literature can be different, for example in Anglés-Alcázar et al. (2017). In their work, "transfer" is considered as all accretion of particles that have been processed in halos other than the main progenitor of halo $i$ by snapshot $n$. This therefore includes the accretion of particles that were processed in (secondary) progenitor halos that merge into $i$. We think it makes more physical sense to group all halos that end up merging by snapshot $n$ and consider particles processed in those as part of "recycling" rather than "transfer". This distinction is quite significant, as the fraction of mass that is processed in non-main progenitors is of the same order of that contributed by the main progenitor.

\section{BARYON AND DARK MATTER BUILD-UP IN HALOES WITH FIDUCIAL PHYSICS}

In this section we focus on analysing the accretion rate of baryons and DM onto haloes in the EAGLE simulation suite, in particular the fiducial physics implementation in L50-REF. We approach this topic with the aim of understanding the differential manner in which baryons and DM are accreted onto haloes, and their main channels of accretion. In $\S 4$, we explore the same problem, focusing instead on changes which can arise based on the feedback models implemented in the simulation. We remark that in this paper, we do not focus on the physical properties of accreted matter (for instance their temperature, density and metallicity), and defer a full investigation of these properties in the context of halo inflow for a future paper.

\subsection{Comparing our algorithm and past literature}

The results to follow, unless otherwise stated, focus on field haloes in the standard resolution EAGLE runs, as outlined 


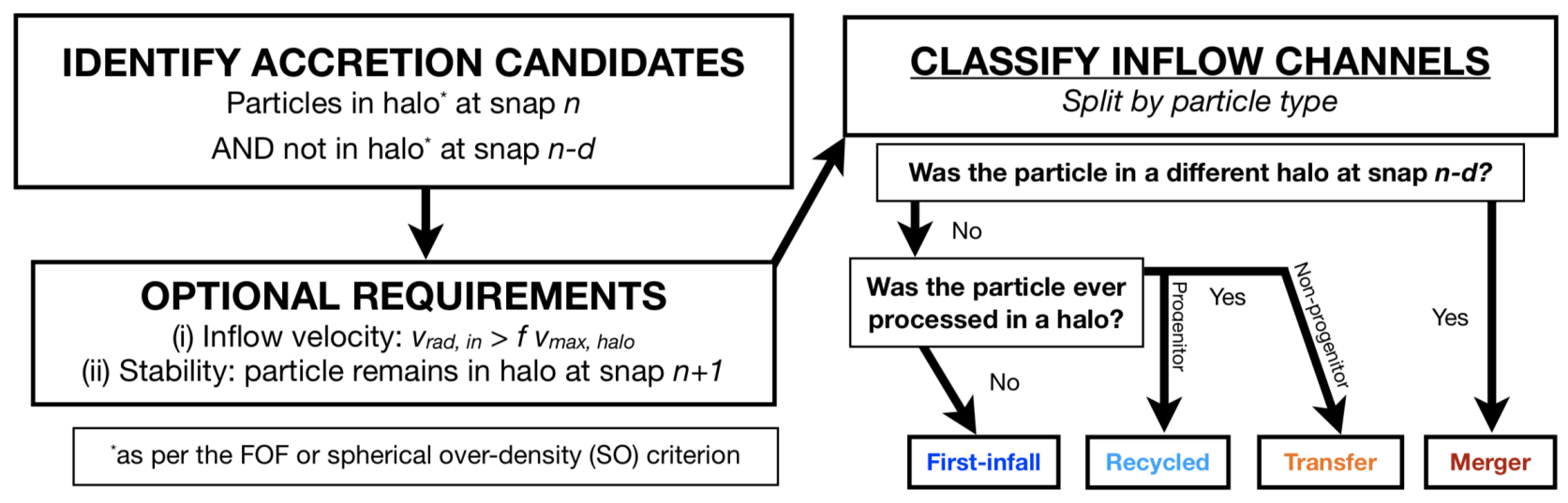

Figure 1. A schematic of our algorithm снимм to calculate accretion rates to haloes. We identify accretion candidates by finding particles which were not in a halo at a snap $n-d$, but joined the halo at snap $n$. We are able to impose a number of extra criteria on these particles before decomposing the total accretion rate into "channels" of accretion based on the processing history of each particle. The code is publicly available at https://github.com/RJWright25/CHUMM.
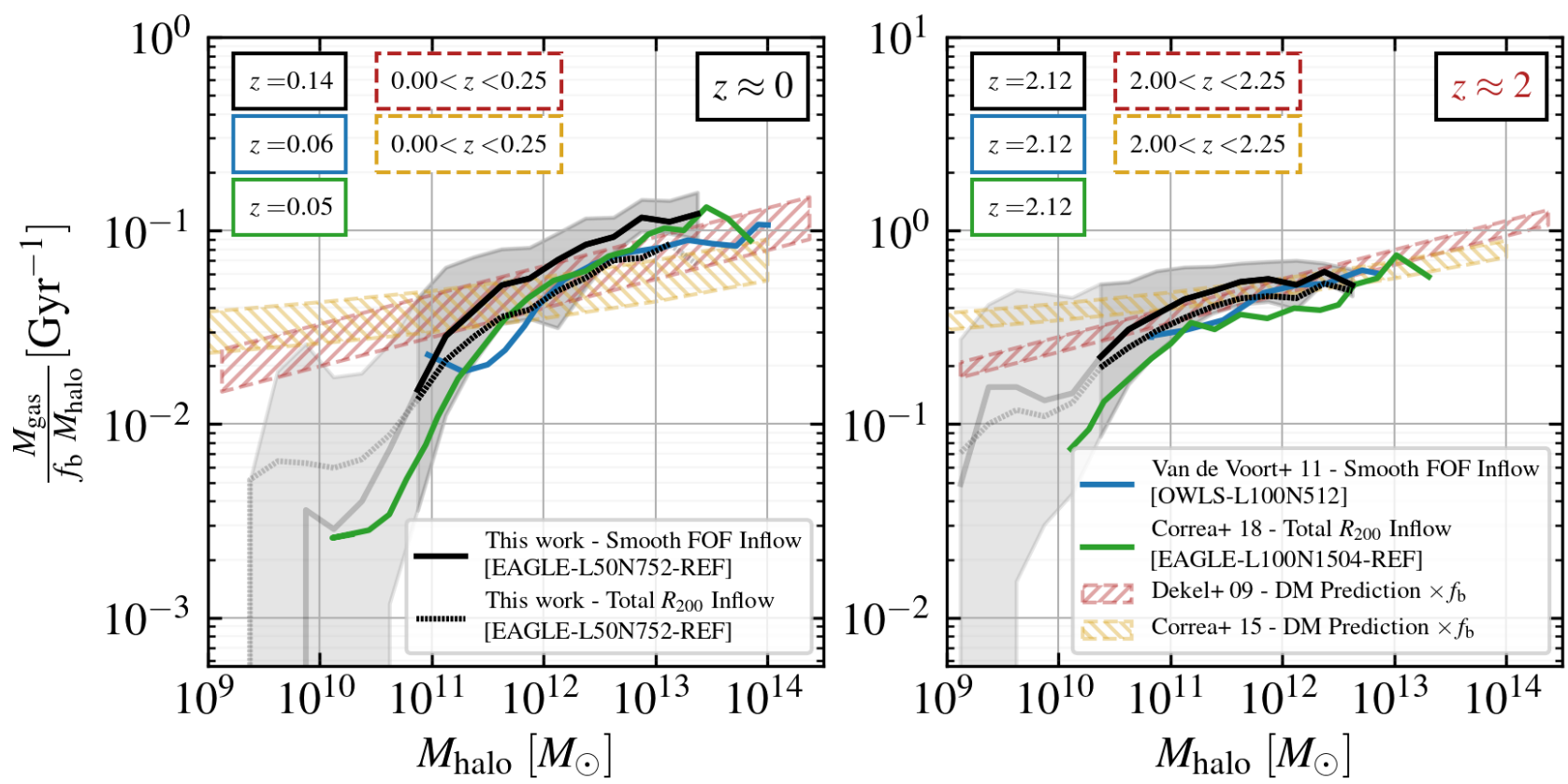

Figure 2. Smooth baryon inflow efficiencies from our algorithm (FOF, solid black lines; and $R_{200}$, densely dotted black lines) for field haloes at $z \approx 0$ (left) and $z \approx 2$ (right), compared to inflow rates from van de Voort et al. (2011), Correa et al. (2018), and those predicted based on DM inflow rates from Dekel et al. (2009) (right-slanting hatched regions) and Correa et al. (2015a,b,c) (left slanting hatched regions). We note that each panel has slightly altered axes limits. Grey shaded regions correspond to the $16^{\text {th }}-84^{\text {th }}$ percentile ranges associated with our L50-REF FOF accretion rate calculation. Line transparency has been increased where the average efficiency has been calculated from a bin in which more than $50 \%$ of haloes were subject to an accretion flux of less than 50 gas particles. We use 24 evenly log-spaced bins of halo mass from $10^{9} M_{\odot}-10^{15} M_{\odot}$, and take the median accretion rate in each of these bins, requiring at least 5 objects to take this average. Our smooth inflow rate refers exclusively to particles not accreted from a different FOF structure. Results from van de Voort et al. (2011) include only smooth accretion, however data from Correa et al. (2018) include the merger channel of accretion. The predictions from Dekel et al. (2009) and Correa et al. (2015a,b,c) (both shown hatched regions, indicating predictions within the given redshift range) are analytic prescriptions of halo mass growth scaled by $f_{b}$ - and as such, also includes merger-based/clumpy accretion. In general, our measurements are qualitatively consistent with previous works, and quantitatively within 0.5 dex for well resolved halo mass bins. 
in Table 1. For an investigation of numerical convergence with higher resolution, we direct the reader to Appendix B. Our primary method of measuring accretion rates uses the FOF inflow algorithm (including accretion onto satellite subhaloes), however we direct the reader to Appendix D for an overview of how using a spherical-overdensity inflow calculation (or, only including accretion onto the central subhalo with the FOF algorithm) would influence our results. Additionally, we attempt to minimise the cuts we impose to our accretion calculations in order to make the results easier to interpret. As such, we stress that in the work to follow, we do not impose any velocity cuts on our results, nor do we impose the stability requirement. We also remark that when we refer to the halo mass of field haloes (associated with FOF inflow), we use the total mass of all particles identified as part of the FOF, not just those within $R_{200}$, crit (with the exception of halo mass in the context of our spherical-overdensity accretion algorithm, for which we use $M_{200}$, crit). For FOF masses below $10^{13} M_{\odot}, M_{200}$, crit corresponds very tightly with $M_{\text {FOF }}$, but for FOF masses of $>10^{13} M_{\odot}, M_{200}$, crit is systematically lower than $M_{\text {FOF }}$ by up to $50 \%$. The method of binning and averaging has significant bearing on the numerical results we present: we indicate the bins we use in the caption of each figure, and only take averages in bins where there are $\geq 5$ objects. For information on the number statistics we use over halo mass, we show the halo mass distributions for relevant runs in Appendix A. Lastly, where possible, we show transparent lines where accretion rates have been calculated from a flux of less than 50 particles.

We first compare our results using the fiducial physics L50-REF box to the previous work of van de Voort et al. (2011), Correa et al. (2018) and the DM-based predictions from Dekel et al. (2009) and Correa et al. (2015a,b,c) in Figure 2. As discussed in $\S 1.2$, the techniques adopted in the past to measure inflow in simulations (and the simulations themselves) are diverse, and it is important to understand the influence of the methodology on inflow measurements. Figure 2 shows gas accretion efficiency (gas accretion rate normalised by halo mass and cosmological $f_{\mathrm{b}}$ ) as a function of halo mass, for redshifts $z \approx 0$ and $z \approx 2$. Our results using the FOF-based algorithm (solid black lines) include only the smooth component accretion (accretion from particles not identified as part of a VELOCIRAPTOR FOF structure at the previous snapshot), while our results using the $R_{200^{-}}$ based algorithm (dotted lines) include particles of all origins. The redshift we quote for our calculations is the mean redshift of the two snaps used to calculate accretion over, and where possible, we quote the redshift of previous works in the same way. As outlined in $\S 1.2$, previously used methods of calculating inflow rates in simulations are diverse - van de Voort et al. (2011) use a FOF halo-based method of identifying inflow particles in the OWLS simulations, while Correa et al. (2018) calculate require mass flux from particles which start outside the FOF and end up inside the virial radius, $R_{200 \text {, crit }}$, in the EAGLE simulations. The results from Correa et al. (2018) include particles of all origins, while the results from van de Voort et al. (2011) include only smooth inflow. We also illustrate the analytic mass-growth predictions from Dekel et al. 2009 and Correa et al. (2015a,b,c) in Figure 2 with dashed lines, corresponding to the total expected halo mass growth scaled by a factor of $f_{\mathrm{b}} \approx 0.16$.
Our results using both the FOF and $R_{200}$ algorithms appear qualitatively consistent with the works of van de Voort et al. (2011) and Correa et al. (2018), with a trend of increasing accretion efficiency with halo mass and redshift. In agreement with these data, compared to the DMbased predictions from Dekel et al. (2009) and Correa et al. (2015a,b,c), we observe significant reduction in gas accretion rates to less massive haloes, below $M_{\text {halo }} \approx 10^{11.5} M_{\odot}$. We see that the FOF algorithm predicts higher accretion rates than the $R_{200}$ method by a steady $\approx 0.15 \mathrm{dex}$, for $M_{\text {halo }} \gtrsim 10^{11.5} M_{\odot}$. We attribute the higher accretion rates in our FOF algorithm compared to the spherical-overdensity method to be principally due to the inclusion of accretion onto satellites subhaloes associated with each of the field haloes. We investigate the difference between accretion onto the central subhalo (excluding satellites) and our primary FOF algorithm (including satellites) in Appendix D, and see that accretion rates to the central subhalo are reduced compared to the primary method by $\approx 0.1$ dex across much of the halo mass range. For the remainder of the paper, we elect to continue to include the accretion onto satellites as our primary method, since we are interested with accretion onto the entire FOF group.

Quantitatively, we see that our FOF method appears to predict slightly higher accretion rates at $z \approx 0$ and $z \approx 2$ by $0.1-0.2$ dex compared to van de Voort et al. (2011) and Correa et al. (2018) (even when the latter results include merger-based accretion). We attribute the difference in our results compared to Correa et al. (2018) to the above discussion of including satellite accretion - and if we only account for accretion onto the central subhaloes, the (already slight) tension with Correa et al. (2018) is greatly reduced. Unsurprisingly, when we use an $R_{200}$ spherical overdensity method (densely dotted lines), we see very good agreement with Correa et al. (2018). Since van de Voort et al. (2011) also use a FOF algorithm, the inclusion of satellite accretion does not explain our increased gas accretion rates compared with their results. van de Voort et al. (2011) do, however, show that using a higher resolution simulation (closer to our primary EAGLE L50-REF box, with a $50 \mathrm{Mpc}$ box with $2 \times 512^{3}$ particles, instead of their primary $100 \mathrm{Mpc}$ box with $2 \times 512^{3}$ particles), that gas accretion rates are enhanced by $\approx 0.1 \mathrm{dex}$ - meaning much of the tension could be attributed to the resolution difference. We also show in Appendix B that using a higher resolution simulation appears to slightly increase our gas accretion rates for low mass haloes with $M_{\text {halo }} \lesssim 10^{11.5} M_{\odot}$. Any further differences are potentially attributable to (i) VELOCIRAPTOR vs. SUBFIND halo catalogues, and (ii) for the $z \approx 0$ panel, the slight differences in measurement redshifts. We also note that we have compared measurements with the work of Mitchell et al. (2020a), and find consistent results over redshift and halo mass.

The comparison presented here gives us confidence that our algorithm is giving results that are largely consistent with previous literature, and can therefore be used for an indepth analysis comparing the rates and channels of baryon and DM accretion onto haloes.

\subsection{Comparing baryon and dark matter accretion}

In this section, we directly compare halo-scale baryonic and DM inflow rates in the EAGLE L50-REF run, showing the 


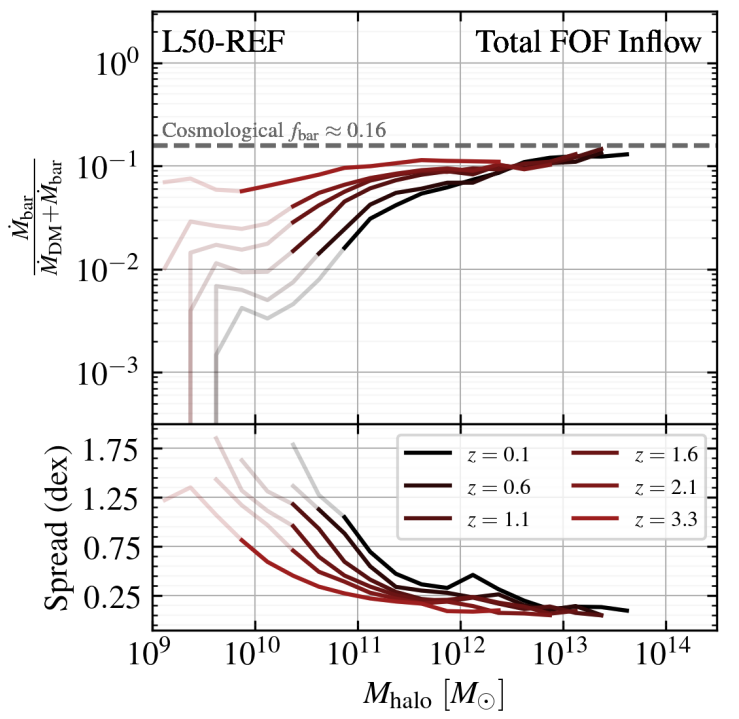

Figure 3. Top panel: the baryon fraction of all halo-accreted matter (inflow baryon fraction, or $f_{b}$, inflow) as a function of halo mass and redshift. Bottom panel: the $16^{\text {th }}-84^{\text {th }}$ percentile range of inflow baryon fraction in dex, as a function of halo mass and redshift. We use 24 evenly log-spaced bins of halo mass from $10^{9} M_{\odot}$ $10^{15} M_{\odot}$, and take the median accretion rate in each of these bins, requiring at least 5 objects to take this average. Line transparency has been increased where the average efficiency has been calculated from a bin in which more than $50 \%$ of haloes were subject to an accretion flux of less than 50 gas particles. For each redshift selected, we see a trend of increasing $f_{\mathrm{b}}$, inflow with halo mass up to $M_{\text {halo }} \approx 10^{12} M_{\odot}$, above which the median fraction plateaus slightly shy of the simulation cosmological baryon fraction. Inflow baryon fractions also universally increase with redshift, the dependence most notable for lower mass haloes, $M_{\text {halo }} \lesssim 10^{11.5} M_{\odot}$. The spread in $f_{\mathrm{b}}$, inflow is largest in the same halo mass range, particularly at low redshift.

influence that the baryon richness of accreting matter can have on resultant halo properties and inflow channels.

\subsubsection{Influence on halo properties}

Above, we discussed the behaviour of gas accretion alone with halo mass and redshift. To directly compare the buildup of baryons and DM in haloes, we investigate the baryon fraction of matter accreted to field haloes in the EAGLE L50-REF run as a function of halo mass and redshift in Figure 3. We calculate the baryon content of accreted matter $\left(f_{\mathrm{b}}\right.$, inflow $)$ for each halo by calculating the summed mass of accreted baryons, $\Delta M_{\mathrm{bar}}$ in, and normalising by the summed mass of all accreted material (DM and baryons),

$f_{\mathrm{b}, \text { inflow }}=\frac{\Delta M_{\mathrm{bar}, \text { in }}}{\Delta M_{\mathrm{bar}, \text { in }}+\Delta M_{\mathrm{DM}, \text { in }}}$.

In the top panel of Figure 3 , we show $f_{\mathrm{b}}$, inflow against halo mass, using total inflow onto field haloes (including both smooth-mode inflow and merger-mode inflow). The baryon inflow rates which may be trivially expected based on DM inflow rates would correspond to $f_{\mathrm{b}}$, inflow $=$

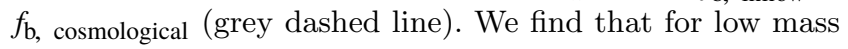

haloes $\left(M_{\text {halo }} \lesssim 10^{12} M_{\odot}\right)$, the composition of inflow material is baryon poor compared to the universal $f_{\mathrm{b}}$ - a finding consistent with the low gas accretion efficiencies in this mass range first presented in van de Voort et al. (2011) (with OWLS) and Correa et al. (2018) (with reference EAGLE physics). At $z \approx 0$, the median baryon fraction of inflow at $M_{\text {halo }} \approx 10^{11} M_{\odot}$ is only $\approx 2 \%$, roughly 10 times lower than the universal baryon fraction $f_{\mathrm{b}} \approx 0.16$, and the halo baryon fractions predicted in Crain et al. (2007). The baryon content of accreted matter increases with halo mass to $f_{\mathrm{b}}$ only for the most massive group sized haloes in the simulation, at $M_{\text {halo }} \gtrsim 10^{13} M_{\odot}$. In addition, the baryon content of accreted matter increases with redshift for low mass haloes, $M_{\text {halo }} \lesssim 10^{11.5} M_{\odot}$, being roughly 1 dex higher at $M_{\text {halo }} \approx 10^{10.5} \widetilde{M}_{\odot}$ at $z \approx 2$ compared to $z \approx 0$. Conversely, for higher mass haloes, $M_{\text {halo }} \gtrsim 10^{11.5} M_{\odot}$, there is little redshift dependence. In the bottom panel of Figure 3, we show the spread (in dex) of the $16^{\text {th }}-84^{\text {th }}$ percentiles in $f_{\mathrm{b}}$, inflow . The spread in $f_{\mathrm{b}}$, inflow is largest for low halo masses - at $z \approx 0$, for $M_{\text {halo }} \approx 10^{11} M_{\odot}$, we see a spread of $1 \mathrm{dex}$, while for $M_{\text {halo }} \approx 10^{13} M_{\odot}$ we see a spread of 0.2 dex. This spread also decreases (for all halo masses) with increasing redshift. We find that the result of suppressed gas inflow (and larger variation in gas inflow) at low halo masses remains clear when we use the higher resolution EAGLE L25N752-RECAL run (see Appendix B), indicating that this result is not a consequence of numerical effects.

This result clearly begs the question: what physics in the simulation drives the depletion of halo baryon inflow, particularly for low mass haloes, $M_{\text {halo }} \lesssim 10^{11.5} M_{\odot}$ ? We investigate the origin of this effect (in the context of feedback) in $\S 4$, where we use various EAGLE runs to investigate the effect on accretion rates in the presence and absence of these different feedback mechanisms. We find that in modern simulations, stellar and AGN feedback mechanisms are strong enough to influence gas dynamics at the halo-scale and modulate inflow rates.

To illustrate the impact that variations in baryon inflow has on the resulting halo population, in Figure 4 we show the baryon fraction of haloes (as defined in Equation 8) as a function of their baryon inflow content, $f_{\mathrm{b}}$, inflow , at both $z \approx 0$ and $z \approx 2$. We restrict the mass range to $10^{10.0} M_{\odot}<$ $M_{\text {halo }}<10^{12.5} M_{\odot}$, where we see the greatest dynamic range in inflow baryon fractions as per Figure 3, still with adequate resolution.

$f_{\mathrm{b}, \text { halo }}=\frac{\left[M_{\mathrm{gas}}+M_{\star}+M_{\mathrm{BH}}\right] \mathrm{FOF}}{M_{\mathrm{tot}, \mathrm{FOF}}}$.

At both redshifts, we observe a tight correlation between $f_{\mathrm{b}}$, inflow and $f_{\mathrm{b}}$, halo, with a roughly constant spread of $\approx 0.3-0.4$ dex (a factor of 2-3) across the range of $f_{\mathrm{b}}$, inflow. The correlation is not linear, and appears to scale more closely with an exponent of 0.5 , i.e. $f_{\mathrm{b}}$, halo $\propto f_{\mathrm{b} \text {, inflow }}^{0.5}$ for both redshifts. While the slope is similar for both redshifts, we see that at $z \approx 2$, the dynamic range in $f_{\mathrm{b}}$, inflow is narrower (as per Figure 3 ), and that $f_{\mathrm{b}}$, halo is systematically higher. If we use a different mass cut and focus on higher mass haloes, $M_{\text {halo }} \gtrsim 10^{12} M_{\odot}$, the correlation remains (with a similar slope) - but over a much smaller dynamic range, from just below to the cosmological $f_{\mathrm{b}}$ for both $f_{\mathrm{b}}$, inflow and $f_{\mathrm{b}}$, halo. This tight correlation indicates that the baryon- 


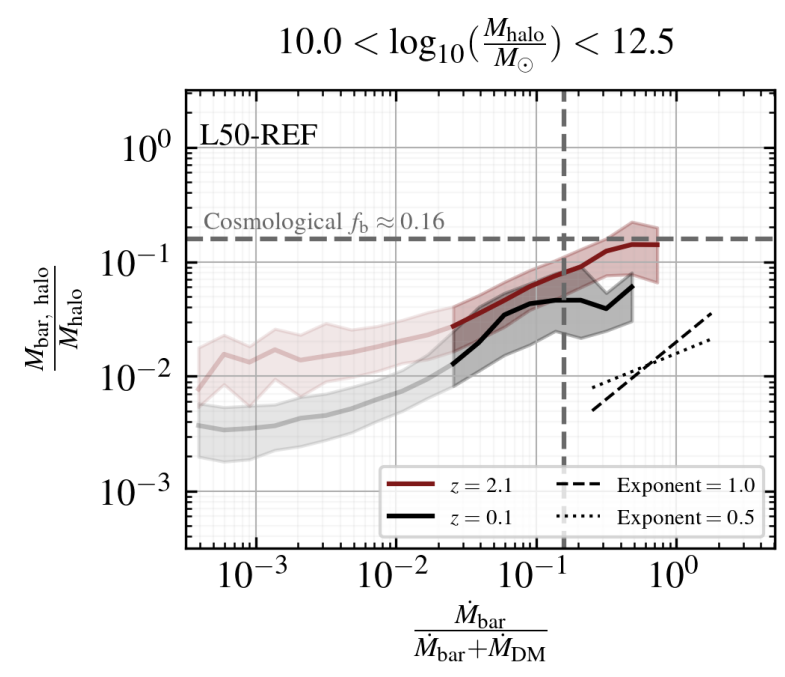

Figure 4. The median halo-baryon fraction (including gas, stellar, and black hole particles in the FOF - see Equation 8) as a function of halo-inflow baryon fraction (see Equation 7), for field haloes in the mass range $10^{10.5} M_{\odot}<M_{\text {halo }}<10^{12.0} M_{\odot}$ at $z \approx 2$ and $z \approx 0$. Line transparency has been increased where the average efficiency has been calculated from a bin in which more than $50 \%$ of haloes were subject to an accretion flux of less than 50 particles. Shaded regions correspond to the $16^{\text {th }}-84^{\text {th }}$ percentile ranges. At both redshifts we observe a tight correlation between the accretion baryon fraction and the halo baryon fraction with $\lesssim 0.5$ dex spread, the correlation becoming slightly steeper at $z \approx 0$. The segments at the right of the panel show for reference a linear dependence and a power law of exponent 0.5. At both redshifts, the slope is shallower than linear, and closer to a power law with an exponent of 0.5 .

richness of a halo is clearly associated with baryon inflow onto that halo - and that if we see variation in baryon inflow at the halo-scale, we would also expect to see this reflected in the baryon content and consequent properties of that halo. The correlation of Figure 4 suggests that haloes can be baryon depleted because the material that gets accreted is baryon poor rather, and not necessarily as a result of baryon processes internal to haloes and galaxies acting to remove the gas. The latter may still occur, but the picture is clearly multi-faceted, with the baryon content of inflow also possibly playing a major role in regulating the baryon content of haloes. Below, we further analyse the connection between the baryon content of haloes with the baryon content of the inflowing matter.

To investigate the distribution of baryons in field haloes and the consequence of the established variations in baryon inflow, in Figure 5 we show the median mass fractions of baryon particles in different intra-halo reservoirs (each row), as a function of their halo mass at $z \approx 0$ (left) and $z \approx 2$ (right). The parameter space in each panel is coloured by the median excess (calculated per halo mass bin) $f_{\mathrm{b}}$, inflow in that parameter range - that is, how baryon rich the inflow is, compared to the median baryon fraction of the inflow at a given halo mass. As a function of halo mass, for each row of panels from top to bottom in Figure 5, we show the mass fractions in: (i) all baryons, (ii) CGM gas (that is, all halo gas excluding star forming gas within the central $30 \mathrm{kpc}$ of the halo), (ii) star-forming ISM gas (star-forming gas within the central $30 \mathrm{kpc}$ of the halo center-of-mass), and (iv) stellar mass (within the central $30 \mathrm{kpc}$ of the halo center-of-mass). Each of these mass fractions are normalised by the total mass of the halo, $M_{\mathrm{FOF}}$.

Focusing on the top row of panels in Figure 5, we display the results seen in Figure 4 as a function of halo mass. We observe that the spread in total halo baryon fraction at fixed halo mass (up to $M_{\text {halo }} \approx 10^{12} M_{\odot}$ ) is very well-correlated with the baryon fraction of accreted matter both at $z \approx 0$ and $z \approx 2$. Specifically, on average, baryon-depleted haloes appear to be host to baryon-depleted inflow, and baryon-rich haloes appear to be host to baryon-rich inflow. At higher halo masses, $M_{\text {halo }} \gtrsim 10^{12} M_{\odot}$, there is far less spread in halo baryon fractions, and most haloes are relatively baryon rich (approaching the cosmological $f_{\mathrm{b}}$ in the grey dashed line). The remaining bottom 3 rows of panels in Figure 5 show where the baryonic matter is distributed in haloes, and also whether instantaneous baryon inflow has impact on the magnitude of these various reservoirs. If we observe a gradient in colour along the spread in mass fraction for a fixed halo mass, it indicates that baryon inflow plays a role in regulating this reservoir over the timescale when accretion was measured.

Panels in the second row of in Figure 5 show the gas fraction in the CGM as a function of halo mass. The CGM gas fraction increases with halo mass, and is the primary baryonic constituent of haloes for those with masses above $M_{\text {halo }} \approx 10^{11} M_{\odot}$. We see that for the majority of the halo mass range (below $M_{\text {halo }} \approx 10^{12.5} M_{\odot}$ ), the spread in CGM mass fraction at fixed mass is strongly correlated with $f_{\mathrm{b}}$, inflow $)$. This is an unsurprising result: one would expect the majority of accreting baryons reside in the CGM at the snapshot subsequent to the accretion measurement. At a halo mass of $M_{\text {halo }} \approx 10^{12} M_{\odot}$, we see approximately 0.5 dex of spread in between the $16^{\text {th }}$ and $84^{\text {th }}$ percentiles. The spread in $f_{\mathrm{b}}$, inflow associated with these percentiles is also approximately $0.5 \mathrm{dex}$ at $z \approx 0$ - telling us that variations in the baryon fraction of the inflowing matter could play a dominant role in driving CGM gas content in reasonably massive haloes. The work of Davies et al. (2019) and Oppenheimer et al. (2020) indicate that variations in black hole mass and activity in EAGLE are associated with variations in CGM gas fractions for Milky-Way like haloes (in the mass range $\left.10^{12} M_{\odot} \lesssim M_{\text {halo }} \lesssim 10^{12.5} M_{\odot}\right)$. In this mass bracket, we show that baryon inflow rates vary strongly over the range of $f_{\text {CGM }}$ values, which show a $16^{\text {th }}-84^{\text {th }} f_{\text {CGM }}$ percentile spread of $\approx 0.5$ dex. Our work suggests a comparitively trivial picture for CGM gas content: in that CGM gas richness is, to first order, a function of baryon accretion rates. These baryon accretion rates are themselves dependent on the specific state of each halo (in particular with respect to baryonic feedback processes, see $\S 4$ ).

In the third row \& fourth row of panels in Figure 5, we show the mass fraction of each halo held in star-forming gas and stellar mass respectively, within the central $30 \mathrm{kpc}$ of a halo. This, to first order, corresponds to the cool/dense phase of the ISM, and the stellar component of the central galaxy, respectively. Over the well-resolved halo mass range, we observe that the spread in the mass fraction contributed by these mass reservoirs is not correlated with $f_{\mathrm{b}}$, inflow. This combination of findings tells us that while the baryon content of inflow has significant bearing on the instantaneous 


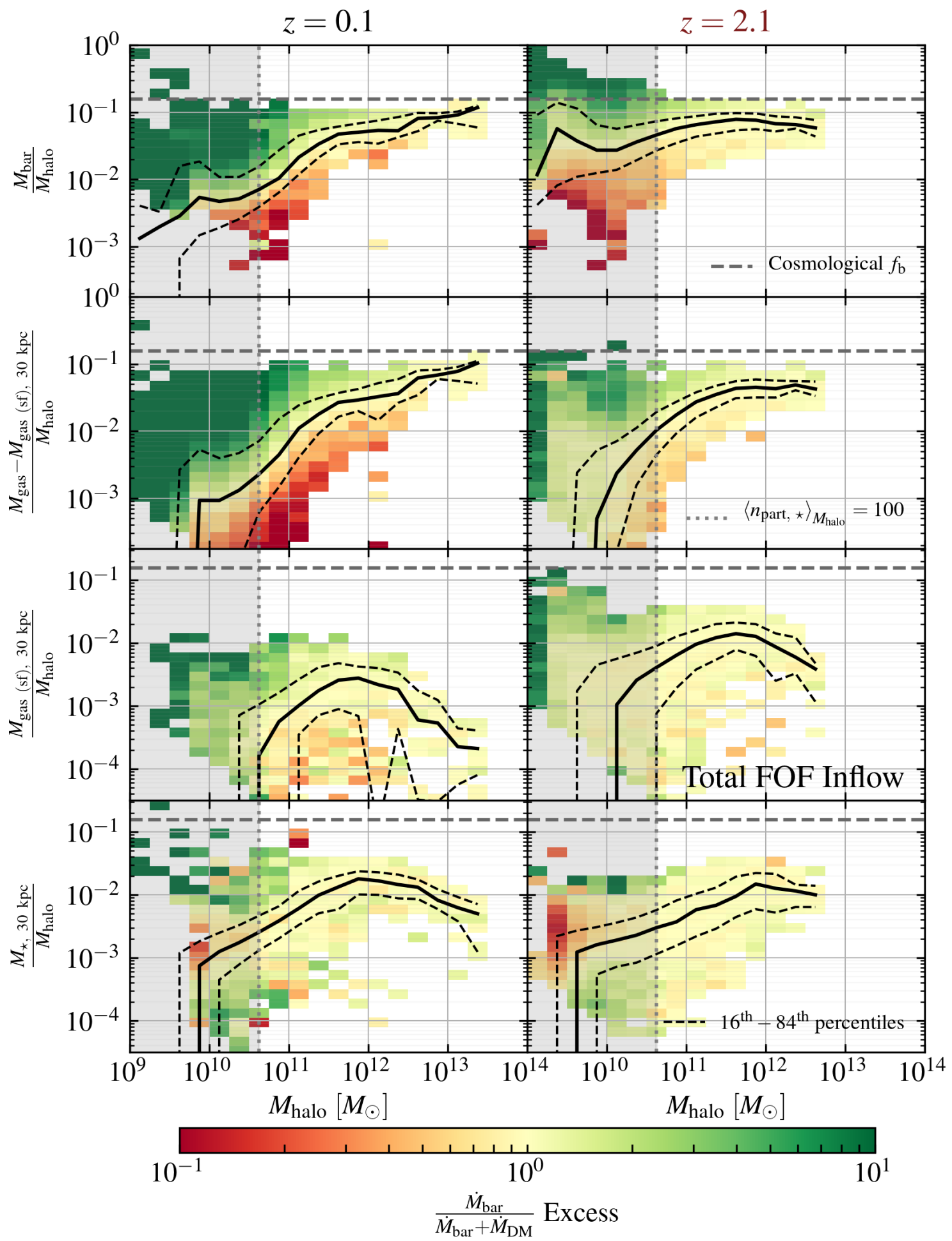

Figure 5. The median fractions of mass contained in various baryon reservoirs, as a function of halo mass for field haloes (left panels: $z \approx 0$, right panels: $z \approx 2$ ). Dashed lines correspond to $16^{\text {th }}-84^{\text {th }}$ percentiles, and the grey shaded regions represents the mass range corresponding to haloes with, on average, less than 100 star particles. We use 24 evenly log-spaced bins of halo mass from $10^{9} M_{\odot}-$ $10^{15} M_{\odot}$, and take the median accretion rate in each of these bins, requiring at least 5 objects to take this average. All panels are coloured by the median excess $f_{b}$, inflow in each halo mass bin. Top panels: the median total baryon fraction of field haloes as a function of halo mass. Second row: The median mass fraction in halo CGM gas (which we define as gas that is not star-forming in the central $30 \mathrm{kpc}$ of a halo). Third row: the median mass fraction in halo ISM gas (star-forming gas within the central $30 \mathrm{kpc}$ of a halo). Bottom panels: the median mass fraction in stars within the central $30 \mathrm{kpc}$ of a halo. We observe that for a fixed halo mass, the spread in total halo baryon fractions is well correlated with the baryon fraction of halo-accreted matter, with baryon depleted (rich) haloes typically being subject to baryon depleted (rich) inflow. We can see this correlation is strongest for the gas outside $30 \mathrm{kpc}$ and for non-star-forming (either warm or insufficiently dense) gas in the central $30 \mathrm{kpc}$. However, the central star-forming gas and stellar component of the halo do not see significant enhancement as a result of instantaneous baryon inflow.

MNRAS 000, 1-25 (2020) 
gas content of the CGM and potentially the warmer phase of central galaxy, over the timescale that we measure accretion $(\approx 1$ Gyr at $z=0)$, the central galaxy's dense ISM and stellar mass content are not immediately influenced by how baryon rich the inflow is.

\subsubsection{Channels of baryonic and DM inflow}

In Figure 6, we compare the inflow channels of baryonic matter (top) to DM (bottom) in across halo mass for redshifts $z \approx 0$ and $z \approx 2$. We split inflow into 4 categories, namely: (i) first infall accretion: that is, inflow particles which had never previously been part of a VELOCIRAPTOR halo structure (blue); (ii) recycled accretion: particles which have been processed in some progenitor halo in the past, but were most recently accreted via smooth-mode cosmological accretion (green); (iii) transfer accretion: particles which have been processed in some non-progenitor halo in the past, but were most recently accreted via smooth-mode cosmological accretion (yellow); or (iv) merger/clumpy accretion, which at the previous snap were part of another halo (red). The sum of channels (i), (ii) and (iii) corresponds to smooth or cosmological accretion - particles which were not in a structure at the previous snapshot. We illustrate the component of baryon accretion contributed by stellar particles with crosshatched regions.

Firstly, regarding the nature of stellar accretion channels, we find that the majority of stellar accretion (in the well-resolved halo mass range) occurs via mergers to high mass haloes, $M_{\text {halo }} \gtrsim 10^{12} M_{\odot}$, though the contribution to lower mass haloes becomes more significant at late times. We remind the reader that we classify infall particles by their type at the snap prior to accretion - but find, on average, that only a very small proportion $(\lesssim 0.1 \%)$ of accreting gas is transformed to star particles at the subsequent snapshot in the halo mass range we analyse. We see significant stellar recycling at the low halo mass end $\left(M_{\text {halo }} \lesssim 10^{11} M_{\odot}\right)$, but given that this corresponds to the regime where haloes contain $\lesssim 100$ stellar particles, we cannot say with certainty that this is not a resolution driven feature.

In general, the breakdown of accretion into its merger and smooth/cosmological (first infall, recycled and transfer) channels is somewhat similar for baryons compared to $\mathrm{DM}$, the notable exception being higher mass haloes at late times, where the merger-mode is significantly more dominant for DM than baryons. At $z \approx 0$, for haloes in the mass range $10^{12.5} M_{\odot}<M_{\text {halo }}<10^{13.5} M_{\odot}$, we observe baryon inflow onto haloes to be $\approx 7 \%$ merger-mode, compared to DM inflow which accumulates $\approx 20 \%$ via merger mode. This tells us that baryon accretion via mergers appears suppressed at late times, comparing to the behaviour we would expect based solely on DM. The physical interpretation of this result is that the infalling haloes contributing to the mergerbased mass growth are already gas poor. We have already seen in Figure 5 that low-mass field haloes are, on average, baryon poor - at $z=0$ containing $\approx 1 \%$ baryons for haloes of mass $M_{\text {halo }} \approx 10^{11} M_{\odot}$, a factor of $>10$ below the universal baryon fraction. Thus, if these baryon-depleted haloes undergo a merger, most of the mass contributed to the descendant will be DM, rather than baryons. If the merger-mass is contributed by infalling satellite subhaloes, such satellites are known to be stripped of their gas in EAGLE via envi- ronmental "pre-processing" prior to infall (see Bahé \& McCarthy 2015; Bahé et al. 2019). Our findings agree well with the work of van de Voort et al. (2011), who found that gas accretion onto haloes is predominantly "smooth-mode", with merger-driven baryon growth significant only in groups and clusters. The contribution from mergers on the DM accretion rate is smaller than the value found in Genel et al. (2010) for the Millennium and Millennium-II Simulations, which they quantified at $\approx 40 \%$ independent of halo mass. This is a factor of $\approx 1.5-4$ times higher than what we find in EAGLE. This is not entirely surprising, as Genel et al. (2010) reached the fraction above by extrapolating the merger rate to large mass ratios, while here we only account for "resolved" halo mergers. It is therefore likely that some fraction of smooth DM accretion corresponds to "unresolved" halo mergers. We believe that some of these "unresolved" halo mergers would correspond to the "recycled" mode of accretion (e.g. if the halo was resolved at some point in the simulation), and some would correspond to "first infall" mode accretion if the halo was never resolved.

We note that the similar breakdown between smooth and merger components of baryon/DM accretion (aside from high mass haloes) is somewhat misleading, and decomposing smooth-mode inflow into its first infall (blue), recycled (green) and transfer (yellow) components illustrates the underlying disparity: we see that accreted DM is significantly more likely to have been pre-processed in haloes than baryons for all redshifts and halo mass bins. The proportion of baryons provided by first infall accretion appears to peak for both redshifts at $M_{\text {halo }} \approx 10^{11} M_{\odot}$ where first infall particles contribute $\approx 60-65 \%$ of all baryon accretion, the peak being slightly more prominent at $z \approx 0$. The contribution of first infall-mode to DM accretion rates is fairly flat with halo mass, at $\approx 25 \%$ for $z \approx 0$ and $\approx 40 \%$ for $z \approx 2$ - both significantly reduced by a factor of $\approx 2$ compared to baryons. The reduced first infall component for DM is met with a marked increase in the recycled inflow component, showing that it is fairly common for DM to be accreted by a halo, ejected from the FOF boundary, and subsequently be re-accreted to a descendant halo. As discussed above, while the classification of inflow into these channels is resolution dependant, we argue that the disparity seen comparing recycled-mode mass growth of baryons and DM is significant. In a future paper, we show that baryon particles in these different channels are indeed characterised by clearly different physical properties (particularly metallicity) and hence, can confidently conclude that our classification of accretion channels are physical, with resolution playing a minor role. We refer the reader to the work of Mitchell et al. 2020a for an analysis of the breakdown of accretion channels as a function of resolution. Relating to our previously measured gas accretion rates in Figures 2 and 3, the picture emerging is that baryons are much less likely than DM to be accreted at all redshifts (and thus, universally, less baryons are classified as processed), particularly for haloes with mass $M_{\text {halo }} \lesssim 10^{11.5} M_{\odot}$.

We see that the contribution of inter-halo transfer accretion across halo mass is small $(\lesssim 5 \%)$ for baryons and DM at both redshifts in the well resolved mass range, in agreement with Mitchell et al. (2020a). The transferred component does appear to increase with halo mass for DM, but remains fairly steady for baryons. The difference in the DM transfer com- 


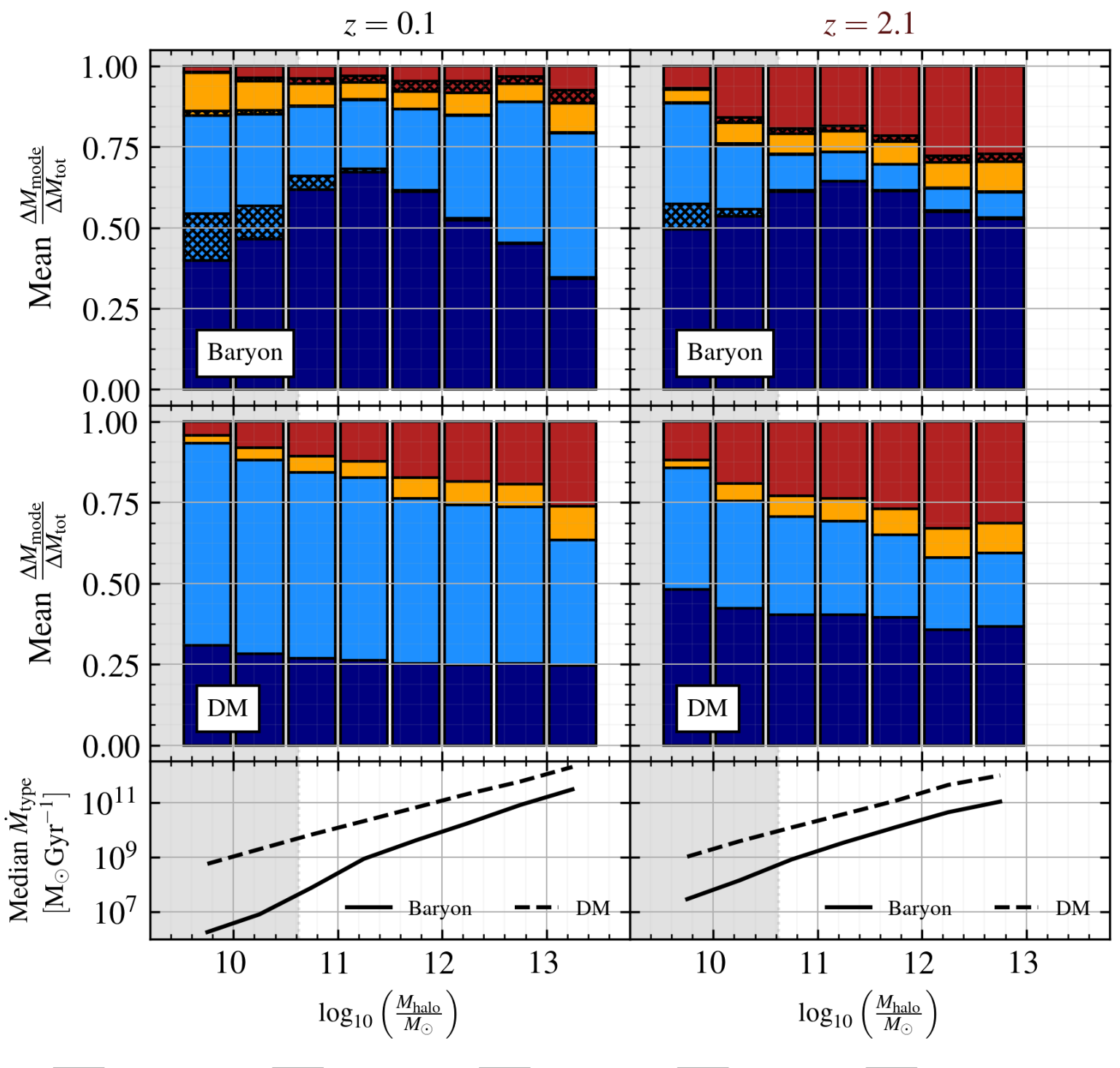

First infall

Recycled

Transfer

Merger

Stellar contribution

Figure 6. Breakdown of the accretion channels of baryons (first row of panels) and DM (2nd row of panels) for $z \approx 0$ (left panels), and $z \approx 2$ (right panels) in the L50-REF run. The results are presented in 8 equally log-spaced $M_{\text {halo }}$ bins from $10^{9.5} M_{\odot}$ to $10^{13.5} M_{\odot}$, and we indicate the halo mass range in which each halo contains less than 100 stellar particles (where numerical issues could influence results) with grey shading. In the bottom panels we also illustrate the median raw accretion rate for each particle type in the same halo mass bins. Accretion channels are categorised as (i) first infall (completely unprocessed particles - blue), (ii) recycled (particles which have channels processed in some progenitor halo in the past, but were most recently accreted via smooth-mode cosmological accretion - green), (iii) transfer (particles which have been processed in some non-progenitor halo in the past, but were most recently accreted via smooth-mode cosmological accretion - yellow), or (iv) (particles which were, at the previous snap, part of another halo - red). We also illustrate the contribution of stellar accretion onto total baryon accretion with black hatched regions in the top panels. The sum of channels (i), (ii) and (iii) corresponds to "smooth-mode" accretion - that is, from particles not in a structure at the previous snapshot. We show the component of accretion contributed by stellar particles with the cross-hatched regions. The difference between DM and baryon accretion channels is most prevalent at late times, with mergers being roughly twice as dominant for DM inflow compared to baryon inflow for all halo mass bins. The proportion of recycled cosmological accretion increases towards late times for both DM and baryonic matter, and at all redshifts we see that DM is much more likely to have been pre-processed prior to accretion compared to accreting baryons, which are more often found to be on first infall to a halo. 
ponent between low- and high-mass haloes is likely due to the fact that low-mass haloes have a shallow potential well that fails to attract DM particles that have left other haloes, and in practice can only re-accrete part of what was originally expelled from the same halo. Consequently, a fraction of the ejected particles from low mass haloes $\left(\lesssim 10^{11} M_{\odot}\right)$, are likely contributing to the "inter-halo transfer" accretion component of the more massive haloes, $>10^{11} M_{\odot}$, as a result of their deeper potential well. It appears that more complex baryonic physics reflects in the transfer component, where we do not see the same obvious variation with halo mass. In $\S 4.2$ we explore this further, and discuss the strong influence that model physics has on baryonic accretion channels.

\subsection{Baryon accretion onto satellite subhaloes}

In this section, we briefly discuss accretion onto satellite subhaloes. Many semi-analytic models of galaxy formation (e.g. Lagos et al. 2018b; Lacey et al. 2016; Henriques et al. 2015) assume that satellite subhaloes are instantaneously cut off from cosmological accretion as soon as they become satellite, subsequently impacting the star formation activity in satellite galaxies. This is an important assumption, and means that many satellite galaxies are very quickly and efficiently quenched in said models. With more realistic hydrodynamical simulations such as EAGLE, it is possible to explore the validity of this assumption.

In the top two panels of Figure 7, we show the median baryon accretion rate for satellite subhaloes as a function of their stellar mass. We note to the reader that the accretion rates to subhaloes that we quote are simply the summed mass of particles which entered a given subhalo between snapshots (irrespective of the origin of each particle). In the top panel, the parameter space is coloured by the binned median stellar-halo mass ratio $\left(M_{\star} / M_{\text {halo }}\right)$. At fixed stellar mass, we see that on average, haloes with higher baryon accretion rates (than expected for their stellar mass) also have a higher stellar-halo mass ratio than their counterparts with lower baryon accretion rates. Wright et al. (2019) showed that the quenching timescales of satellite galaxies in EAGLE were strongly correlated with the ratio between their stellar mass to their host halo mass. This tells us that the physical effect driving the lengthening of the quenching timescale in satellites is likely continuing gas accretion, which is larger in satellites that are massive relative to their host halo (leading to quenching timescales of $\gtrsim 5$ Gyr in the most massive satellites, see Wright et al. 2019). In the second panel of Figure 7, the parameter space is coloured by the binned median halo-centric distance of a satellite to its host's center-of-mass, normalised by the host's virial radius: $\left(\left|\mathbf{r}_{\text {com, sat }}-\mathbf{r}_{\text {com, host }}\right|\right) / R_{200 \text {, host }}$. At fixed stellar mass, we see that the satellites with the greatest baryon accretion rates are, on average, further from their host than those subhaloes with relatively low baryon accretion rates. This tells us that baryon accretion onto the haloes of satellites appears to be suppressed when the satellites fall deep into the host's potential.

While we find there are conditions under which satellite gas accretion can be suppressed, it is important to note that we find satellite subhaloes can continue to accrete baryons, and they are not completely cut-off from cosmological accretion (as many SAMs assume). It is obvious that this

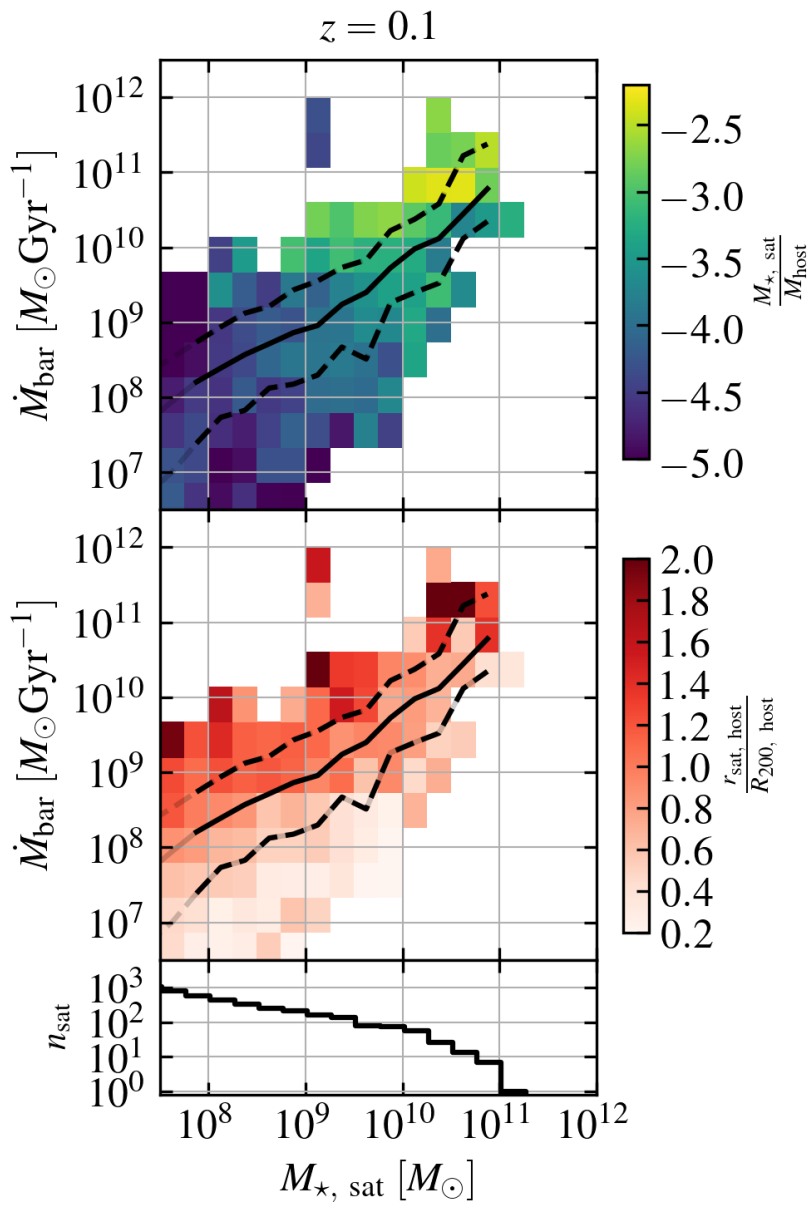

Figure 7. Top panel: The median baryon accretion rate to satellite subhaloes as a function of their galaxy's stellar mass, coloured by the binned median stellar-halo mass ratio: $\frac{\boldsymbol{M}_{\star} \text {, sat }}{\boldsymbol{M}_{\text {host }}}$. Second panel: The median baryon accretion rate for satellite subhaloes as a function of their stellar mass, coloured by the binned median halo-centric distance of a satellite to its host's center-of-mass, normalised by the hosts virial radius: $\left(\mid \mathbf{r}_{\mathrm{com}}\right.$, sat $\left.-\mathbf{r}_{\text {com, host }} \mid\right) / R_{200}$, host . Bottom panel: the number of satellite subhaloes included in each mass bin. Line transparency has been increased where the average efficiency has been calculated from a bin in which more than $50 \%$ of haloes were subject to an accretion flux of less than 50 gas particles. We use 20 bins in stellar mass between $M_{\star}=10^{7} M_{\odot}$ and $M_{\text {star }}=10^{12} M_{\odot}$. At fixed stellar mass, we see that the satellites with the greatest baryon accretion rates are, on average, further from their host than those subhaloes with relatively low baryon accretion rates.

has fundamental implications for the galaxies inhabiting these subhaloes, and their subsequent evolution. We defer a more complete discussion of accretion onto satellite subhaloes (particularly, under what circumstances satellites are net accreting baryonic matter), and the detailed relationship between satellite quenching and the different accretion channels for a future paper. 


\section{ACCRETION AS A FUNCTION OF MODEL PHYSICS}

In this section, we build on $\S 3$, and focus on analysing the same topics when changes are made to feedback physics. In particular, we explore the qualitative and quantitative differences in accretion onto haloes that arise with the inclusion and parameterisation of stellar and AGN feedback.

\subsection{Comparing baryon and dark matter accretion}

In Figure 8, we show the median smooth baryon accretion efficiencies in 2 bins of halo mass over the redshift range $z \approx 0$ to $z \approx 5$, for various feedback implementations as outlined in Tables 1 and 2. This illustrate the main trends in gas accretion efficiency that we observe when changing feedback physics over cosmic time (which we explore further in Figure 9, comparing baryon and DM accretion rates directly). The top panels show the raw gas accretion efficiency over cosmic time, while the bottom panels illustrate the gas accretion efficiency at each redshift, normalised by the gas accretion efficiency seen in the L50-REF run. The left panels in Figure 8 focus on the halo mass range $10^{10.5} M_{\odot}<M_{\text {halo }}<10^{11.5} M_{\odot}$ - the range in which we observe the largest differences with stellar feedback physics, compared to non-radiative and no-feedback physics. In these panels we illustrate the runs in which changes to stellar feedback (or lack thereof) have been made: L50-REF, L32-NONRAD (our in-house GADGET box), L25-NONRAD, L25-NOFB, L50-NOAGN, L25-WEAKSN and L25-STRONGSN). The right panels focus on the halo mass range $10^{12.5} M_{\odot}<M_{\text {halo }}<10^{13.5} M_{\odot}$ - the range in which we observe the largest differences with varying AGN feedback physics. In these panels, we illustrate the runs in which changes to AGN feedback (or lack thereof) have been made: L50-REF, L50-NOAGN, L50-AGNdT8, and L50-AGNdT9. We also compare our work to the DM-based predictions presented in Dekel et al. (2009) (see Equation 2), and Correa et al. (2015a,b,c). These mass scales correspond to either side of the transition mass scale $\left(M_{\text {halo }} \approx 10^{12} M_{\odot}\right)$ described in Bower et al. (2017) - above which star formation driven outflows cease to be buoyant, and are unable to prevent central mass build-up (which ultimately feeds BH mass accretion and AGN feedback).

Before looking at the influence of stellar and AGN feedback, we draw the reader's attention to the differences between the L25-NONRAD (yellow) run and the L25-NOFB (green) run in the left panels of Figure 8 - for the mass range $10^{10.5} M_{\odot}<M_{\text {halo }}<10^{11.5} M_{\odot}$. We notice that while the non-radiative and no-feedback runs follow the same functional form over redshift (overpredicting baryon accretion rates compared to the full physics L50-REF run towards low redshift), the L25-NOFB run exhibits systematically lower baryon inflow rates than the L25-NONRAD run by a factor of $\approx 25 \%$ (or $0.1 \mathrm{dex}$ ) for $z \lesssim 2$. We attribute this to the buildup of particles around the FOF boundary for haloes in the L25-NONRAD run, and the subsequent stochastic crossing and ejection from this boundary adding to accretion events. This is not the case in the L25-NOFB run, where we initially see higher accretion rates due to less thermal pres- sure with the inclusion of radiative cooling (at $z \gtrsim 2.5$ ), but less buildup and stochastic FOF accretion events at later times. To verify this reasoning, we tested imposing our "stability" criterion: where we require particles to remain in the halo at the snapshot after accretion onto be considered a legitimate inflow candidate. When we impose this requirement, we observe that inflow rates in the L25-NOFB and L25-NONRAD runs were essentially identical, supporting our reasoning for the enhanced accretion in L25-NONRAD in Figure 8.

Moving towards the influence of stellar feedback, we find that there is tension between the Dekel et al. (2009) and Correa et al. (2015a,b,c) predictions and the L50-REF run at lower halo mass $\left(10^{10.5} M_{\odot}<M_{\text {halo }}<10^{11.5} M_{\odot}\right)$ particularly at late times, with our accretion efficiencies significantly $(\approx 0.6$ dex on average) lower than that those predicted (as also observed in Figure 2. Since the accretion efficiencies in the L25-NONRAD, L32-NONRAD and L25-NOFB runs appear to agree very well with the DM-based predictions (within a factor of $\approx 2$ ), we argue that the implementation of baryonic feedback processes, in particular stellar feedback, is responsible for the tension at the low-mass end. Moreover, since we also observe the tension with DM-based predictions in the L50-NOAGN run, we argue that it is specifically the inclusion of stellar feedback which causes baryon inflow onto low mass haloes to be severely suppressed (by up to $\approx 1$ dex), agreeing with the arguments of van de Voort et al. 2011 with the older OWLS simulations. The physical origin of this result is outlined in the work of Bower et al. (2017), who show for haloes of mass $M_{\text {halo }} \lesssim 10^{12} M_{\odot}$ that star formation driven outflows are more buoyant than any tenuous corona surrounding the halo, ultimately suppressing gas inflow. This also agrees with the work of Mitchell et al. (2020b), who find that the halo-scale mass loading factor, $\eta=\left\langle\dot{M}_{\text {wind, halo }}\right\rangle /\left\langle\dot{M}_{\star}\right\rangle$, is highest in this mass range, as a consequence of stellar feedback driven outflows. If we increase the energy injected by stellar feedback (as in the L25STRONGSN run, dark blue), we see that gas accretion efficiencies are suppressed by $\approx 20-30 \%$ compared to L50-REF for the full redshift range, tapering slightly towards $z=0$. Correa et al. (2018) illustrate a similar result at the galaxyscale in the L25-STRONGSN run, where gas accretion was also suppressed for central galaxies with $M_{\text {gas }} \lesssim 10^{12} M_{\odot}$. Somewhat counter-intuitively, if we instead tune down the energy injection from stellar feedback (L25-WEAKSN, light blue), then we see a slight suppression in gas accretion rates towards $z=0$ (a result also found at the galaxy-scale by Correa et al. 2018). This highlights the importance of considering the interplay between stellar and AGN feedback - particularly how stellar feedback affects SMBH behaviour. When stellar feedback is weak, SMBHs in EAGLE are allowed to grow more due to the accumulation of gas in galactic central regions, leading to stronger AGN feedback in low-mass haloes (Bower et al. 2017). As such, we argue that the slight decrease in gas accretion seen in L25-WEAKSN is driven by more (unphysically) efficient AGN relative to the L50-REF run.

Focusing now on the right hand panels (where we use a higher mass bin, $10^{12.5} M_{\odot}<M_{\text {halo }}<10^{13.5} M_{\odot}$ ) we concentrate on AGN driven variations in accretion rates. The reader should note that we only test the influence of AGN feedback by using runs with altered temperature boost val- 


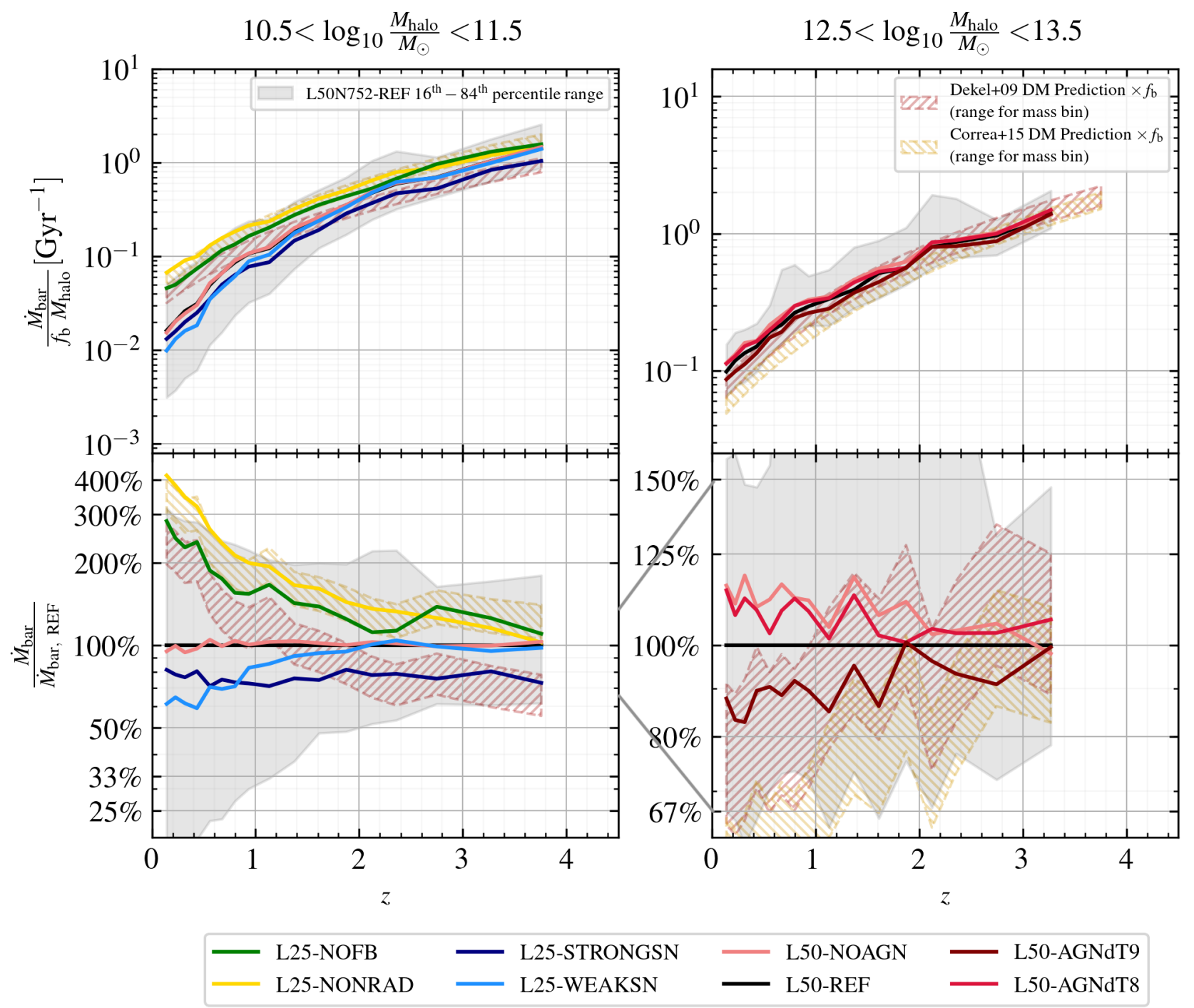

Figure 8. Top panels: Median total (both smooth and merger-based) baryon accretion efficiencies in bins of halo mass $\left(\right.$ left: $10^{10.5} \boldsymbol{M}_{\odot}<$ $M_{\text {halo }}<10^{11.0} M_{\odot}$, right: $\left.10^{12.5} M_{\odot}<M_{\text {halo }}<10^{13.5} M_{\odot}\right)$ over the redshift range $z=0$ to $z=4$, for runs with various physical models, outlined in Tables 1 and 2 (EAGLE reference physics in black, otherwise solid coloured lines). Bottom panels: The median total (both smooth and merger-based) baryon accretion efficiencies for each run, normalised by the baryon accretion efficiency of the L50-REF run. We display the $16^{\text {th }}-84^{\text {th }}$ percentile range of accretion efficiencies for the L50-REF run as the shaded grey region. Our work is also compared to the DM-based prediction presented in Dekel et al. (2009) and Correa et al. (2015c) (hatched pink and yellow, respectively, shaded regions for each mass range). We draw the reader's attention to the different axis ranges in each of the bottom panels. In the lower halo mass range (left) we focus on stellar feedback implementations. We see that compared to the L25-NONRAD and L25-NOFB runs, once stellar feedback is implemented (either in the L50-NOAGN run or the L50-REF run) there is a significant drop in baryon accretion onto haloes, particularly at low redshift (where we see differences of $\approx 300-400 \%$ ). In the higher halo mass range (right) we focus on AGN feedback implementations, where it is clear that removing AGN feedback or using a more efficient AGN feedback model can alter baryon accretion efficiencies at late times by $\approx 20-30 \%$.

ues, essentially controlling how "explosive" the AGN feedback is: meaning we do not consider alterations to the "strength" of AGN (i.e., the energy injection rate). Our work (with fiducial physics) and the DM-based predictions agree to within a factor of 2 in this higher mass regime, and show the same functional form across redshift. This is contextualised by the results in $\S 3$, where we show with fiducial physics that baryon accretion rates approach DM accre- tion rates (scaled to a factor of $f_{\mathrm{b}}$ ) in higher mass haloes, $M_{\text {halo }} \gtrsim 10^{11.5} M_{\odot}$. Focusing on the bottom right-hand panel in Figure 8, we note that variations in AGN feedback make a small difference to the baryon accretion rate compared to the L50-REF box. At $z \approx 0$ relative to the L50-REF run, in the L50-NOAGN run, we observe enhanced gas accretion efficiencies by $\approx 30 \%$, and in the L50-AGNdT 8 run (reduced AGN heating temperature from $10^{8.5} \mathrm{~K}$ in the ref- 
erence box to $10^{8} \mathrm{~K}$ ), we observe an enhancement of closer to $\approx 20 \%$. Conversely, increasing the AGN heating temperature (to $10^{9} \mathrm{~K}$, L50-AGNdT9) induces a decrease in gas accretion efficiencies by $\approx 20 \%$ at $z \approx 0$.

Thus, in EAGLE, we see that the inclusion of stellar feedback can modulate accretion efficiencies in the halo mass range $10^{10.5} M_{\odot}<M_{\text {halo }}<10^{11.5} M_{\odot}$ by a factor of $\approx 4$; while the inclusion and explosivity of AGN feedback can modulate accretion efficiencies in the halo mass range $10^{12.5} M_{\odot}<M_{\text {halo }}<10^{13.5} M_{\odot}$ by a factor of $20 \%-30 \%$. The much larger effect stellar feedback has relative to AGN feedback in these two regimes is due in part to the former acting on average on lower-mass haloes, whose potential wells are shallower than the massive haloes in which AGN feedback acts. We do not investigate the influence of AGN feedback on a halo-to-halo basis, however we refer the reader to the work of Davies et al. (2019) and Oppenheimer et al. (2020) who directly quantify the prevalence of AGN activity in individual galaxies, and show that stronger SMBH activity is correlated with poor CGM gas content. Another important consideration is the buoyant way in which stellar feedbackdriven outflows expand inside haloes, which lead to significant halo gas mass being swept-up and removed from haloes. Mitchell et al. (2020b) showed that halo-scale stellar feedback driven outflows in EAGLE are characterised by a mass loading factor $\approx 10$ times greater than at the galaxy level.

In Figure 9, similar to Figure 3, we illustrate the baryon fraction of smooth-mode halo-accreted matter for redshifts $z \approx 0$ and $z \approx 2$ for our full collection of standard resolution EAGLE variants, outlined in Table 1, as well as our in-house GADGET L32-NONRAD run (Table 2). We also plot the ratio of $f_{\mathrm{b}}$, RUN $/ f_{\mathrm{b}}$, L50-REF in the bottom panels, to better illustrate the variations in each run compared to reference physics. We first focus on the effects of stellar feedback implementation, showing $f_{\mathrm{b}}$, inflow values from the L50-REF model, as well as the weak (L25-WEAKSN, light blue) and strong (L25-STRONGSN, dark blue) stellar feedback runs. For halo masses $M_{\text {halo }} \lesssim 10^{11.5} M_{\odot}$, the L50-REF and L50-NOAGN runs (i.e., runs including stellar feedback) show a significant decrease in $f_{\mathrm{b}}$, inflow compared to the L32-NONRAD, L25-NONRAD and L25-NOFB runs by up to $\gtrsim 300 \%$, with the discrepancy increasing towards low redshift.

At higher redshift $(z \approx 2$, right panels), in the halo mass range $M_{\text {halo }} \lesssim 10^{11.5} M_{\odot}$, we see that decreasing the strength of stellar feedback increases the baryon fraction of inflow by $\approx 20 \%-30 \%$ compared to the L50-REF run, and that increasing the strength of stellar feedback decreases the baryon fraction of inflow by $\approx 40 \%-50 \%$ compared to the L50REF. Interestingly, above this mass range, we see a very slight $(10 \%-20 \%)$ decrease in $f_{\mathrm{b}}$, inflow for both the L25STRONGSN and L25-WEAKSN boxes. Here, we again begin to note the importance of understanding the interplay between stellar and AGN feedback in order to accurately interpret these results (see Bower et al. 2017 for an in-depth analysis of how stellar feedback modulates AGN activity in EAGLE). In the case of the L25-STRONGSN box, we attribute the drop in baryon accretion directly to the stronger stellar feedback (similar to the lower halo mass range). In the L25-WEAKSN box (where one might expect baryon inflow onto be bolstered, rather than suppressed), we attribute the drop to unphysically enhanced AGN feedback, in the ab- sence of sufficiently strong stellar feedback. Physically, less pressure support and heating from stellar feedback allows more gas to be funnelled towards the center of haloes which are host to a SMBH. This increases the $\mathrm{BH}$ accretion rate (and, therefore, the energy injection rate from AGN feedback), causing strong outflows in haloes that in the reference run were only affected by stellar feedback. The extreme case is shown in the L25-ONLYAGN run, where the lack of stellar feedback appears to increase the ability of AGN feedback to suppress gas inflow at low halo masses (down to $\left.M_{\text {halo }} \approx 10^{10.5} M_{\odot}\right)$.

Moving towards lower redshift $(z \approx 0)$, we see that the differences between the L25-STRONGSN and L50-REF runs in the mass range $M_{\text {halo }} \lesssim 10^{11.5} M_{\odot}$ are similar to those analysed above at $z \approx 2$. This said, at $z \approx 0$, we actually measure enhanced baryon accretion in the L25-STRONGSN box compared to L50-REF for haloes of mass $M_{\text {halo }} \gtrsim 10^{11.5} M_{\odot}$. Interestingly, in this mass range, the behaviour of the L25STRONGSN run and the L50-NOAGN run appear almost identical. We argue that this is due to the increased energy injection from stellar feedback causing SMBHs to have decreased accretion rates, eventually leading to suppressed AGN feedback. Hence, in this mass range, doubling stellar feedback energy injection induces the same baryon accretion behaviour we would expect if there were no AGN feedback whatsoever. Correa et al. (2018) show that in the L25-STRONGSN run the gas accretion at the galaxy-scale increased relative to the L50-REF run for galaxies with $M_{\text {gas }} \gtrsim 10^{12} M_{\odot}$. This again points to variations in the gas accretion rate at the galaxy-scale to be largely driven by the variations in the baryon content of the matter accretion at the halo level.

Focusing instead on the effects of varying the AGN feedback implementation (comparing L50-REF, L50-NOAGN, L50-AGNdT8 and L50-AGNdT9), we see for both redshift panels that there is little change below $M_{\text {halo }} \approx 10^{11.5} M_{\odot}$. At $z \approx 2$, we see differences between these runs only for the highest mass haloes, $M_{\text {halo }} \gtrsim 10^{12} M_{\odot}$. Omitting AGN feedback (L50-NOAGN, pink) may modestly increase baryon accretion by $\approx 10 \%-20 \%$ compared to the L50-REF run, and decreasing the injection temperature to $10^{8} \mathrm{~K}$ (from $10^{8.5} \mathrm{~K}$ in the reference physics model) in the L50-AGNdT8 run (red) has the same effect of modestly increasing the baryon inflow (albeit to a slightly lesser degree). Increasing the injection temperature to $10^{9} \mathrm{~K}$ in the L50-AGNdT9 run (maroon) decreases the baryon inflow efficiency by the same very modest percentages.

Towards $z \approx 0$, the same variations in AGN treatment produce much larger variations in baryon inflow. We see the largest amplitude of modulation in the halo mass range $10^{12} M_{\odot} \lesssim M_{\text {halo }} \lesssim 10^{13} M_{\odot}$ - where the omission of AGN feedback increases $f_{\mathrm{b}}$, inflow relative to L50-REF by $\approx 70 \%-80 \%$; decreasing the AGN injection temperature increases $f_{\mathrm{b}}$, inflow by $\approx 30 \%-40 \%$ compared to L50-REF; and increasing the AGN injection temperature decreases $f_{\mathrm{b}}$, inflow by $\approx 30 \%$. Interestingly, for the highest mass haloes in the $50 \mathrm{Mpc}$ boxes at $M_{\text {halo }} \gtrsim 10^{13} M_{\odot}$, AGN physics seems to have very little impact on baryon accretion. Lagos et al. (2018a) show that high mass centrals $\left(M_{\star} \gtrsim 10^{11.8} M_{\odot}\right.$, corresponding to haloes of mass of $\left.M_{\text {halo }} \gtrsim 10^{13} M_{\odot}\right)$ seem to be preferentially more star-forming and fast-rotating than observational counterparts, indicating that AGN feedback 


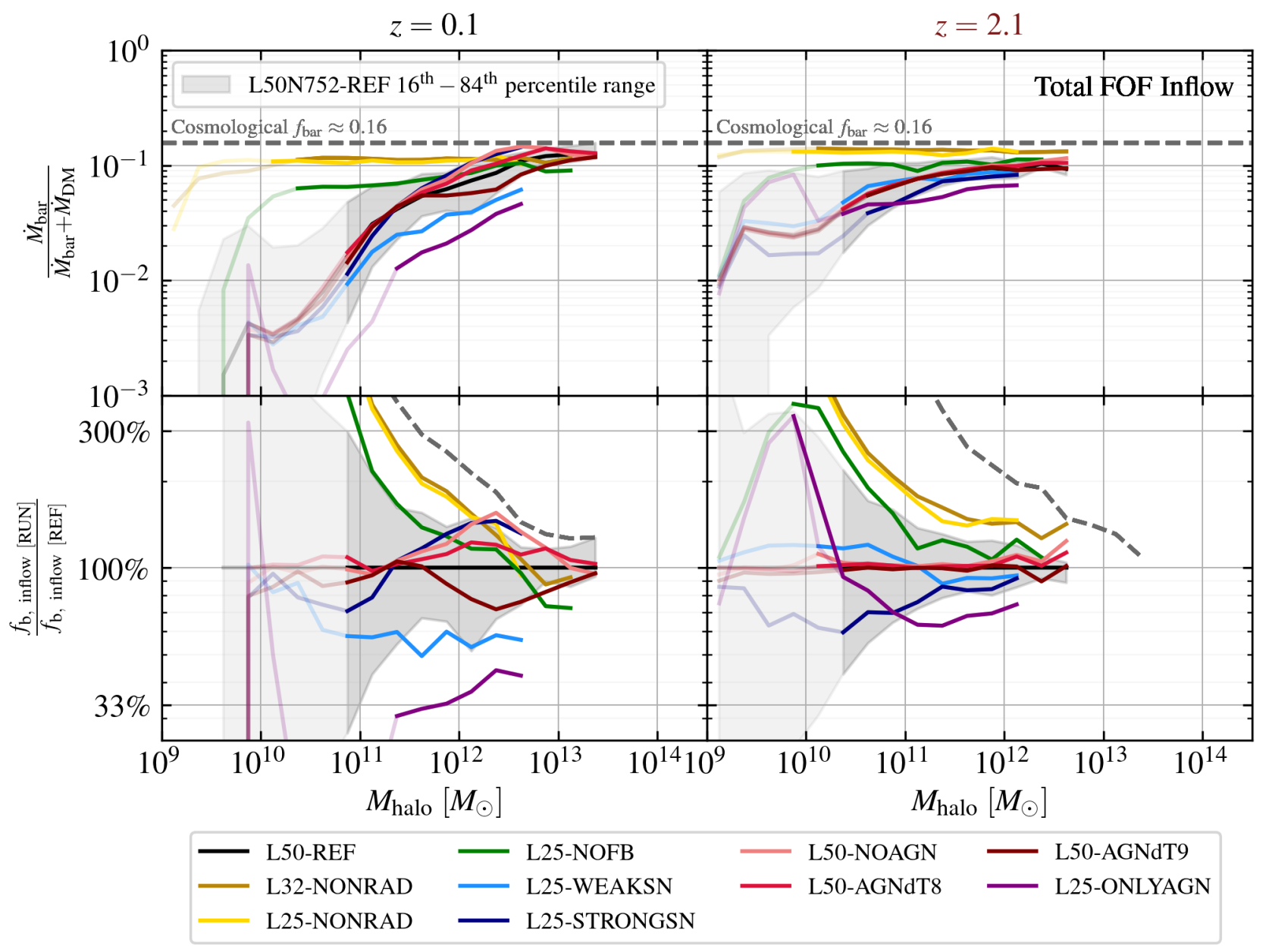

Figure 9. Top panels: The baryon fraction of field halo-accreted matter as a function of halo mass for redshifts $z \approx 0$ (left) and $z \approx 2$ (right) for EAGLE runs with various physical implementations, outlined in Table 1 (reference physics in black, otherwise solid coloured lines). Bottom panels: The baryon fraction of field halo-accreted matter, normalised by the baryon fractions obtained in the L50-REF run. Line transparency has been increased where the average efficiency has been calculated from a bin in which more than $50 \%$ of haloes were subject to an accretion flux of less than 50 particles. To varying extents (and with different functional forms), we see a trend of increasing halo-accreted matter baryon fractions with halo mass for each EAGLE run, with the exception of the adiabatic run which has a roughly constant inflow baryon fraction with halo mass. We see stellar feedback primarily influences the low halo mass population, $M_{\text {halo }}<10^{11.5} M_{\odot}$, while AGN feedback influences the high halo mass population, $M_{\text {halo }}>10^{11.5} M_{\odot}$ (particularly at low redshift).

in the most massive EAGLE systems may not be strong enough. Our findings provide context for this result, with there being little modulation of halo-scale inflow by AGN feedback in the L50-REF run compared to the L50-NOAGN run for massive haloes, $M_{\text {halo }} \gtrsim 10^{13} M_{\odot}$.

\subsection{Channels of baryon and DM inflow}

We now move on to discuss the effect that baryonic physics has on the breakdown of accretion channels across cosmic time. Figure 10 shows the breakdown of accretion channels for baryons and DM in a selection of the EAGLE variations as a function of halo mass, at $z \approx 0$ and $z \approx 2$. In the first row of panels, we show the DM accretion channels from L50-REF, noting that the breakdown of DM accretion channels were effectively identical for each run. From the second row of panels down, show baryon accretion channels for (i) L25-NONRAD, (ii) L25-NOFB, (iii) L50-NOAGN, and (iv)
L50-REF - effectively step-wise introducing new baryonic physics. The bottom panels act to remind the reader of the normalisation of baryon accretion efficiencies in each mass bin for the various runs.

Starting with the non-radiative physics run (L25NONRAD, 2nd row of panels), we see that the breakdown of baryon accretion is very similar to that of DM in L50-REF for each of the halo mass bins. The rate of mergers is highest for massive haloes at high redshift, approaching $35-40 \%$ of all mass accretion at $z \approx 2$. The proportion of recycled accretion increases at late times to a steady $\approx 40-50 \%$, decreasing modestly with halo mass where mergers play a more dominant role. In the 3rd row of panels, we show the influence of introducing radiative cooling on the breakdown of accretion channels with the L25-NOFB run. This change alters the picture significantly, most notably reducing recycling in haloes with mass $M_{\text {halo }} \gtrsim 10^{11} M_{\odot}$ (particularly at $\left.z \approx 2\right)$. It is this change that appears to produce the peak in first in- 


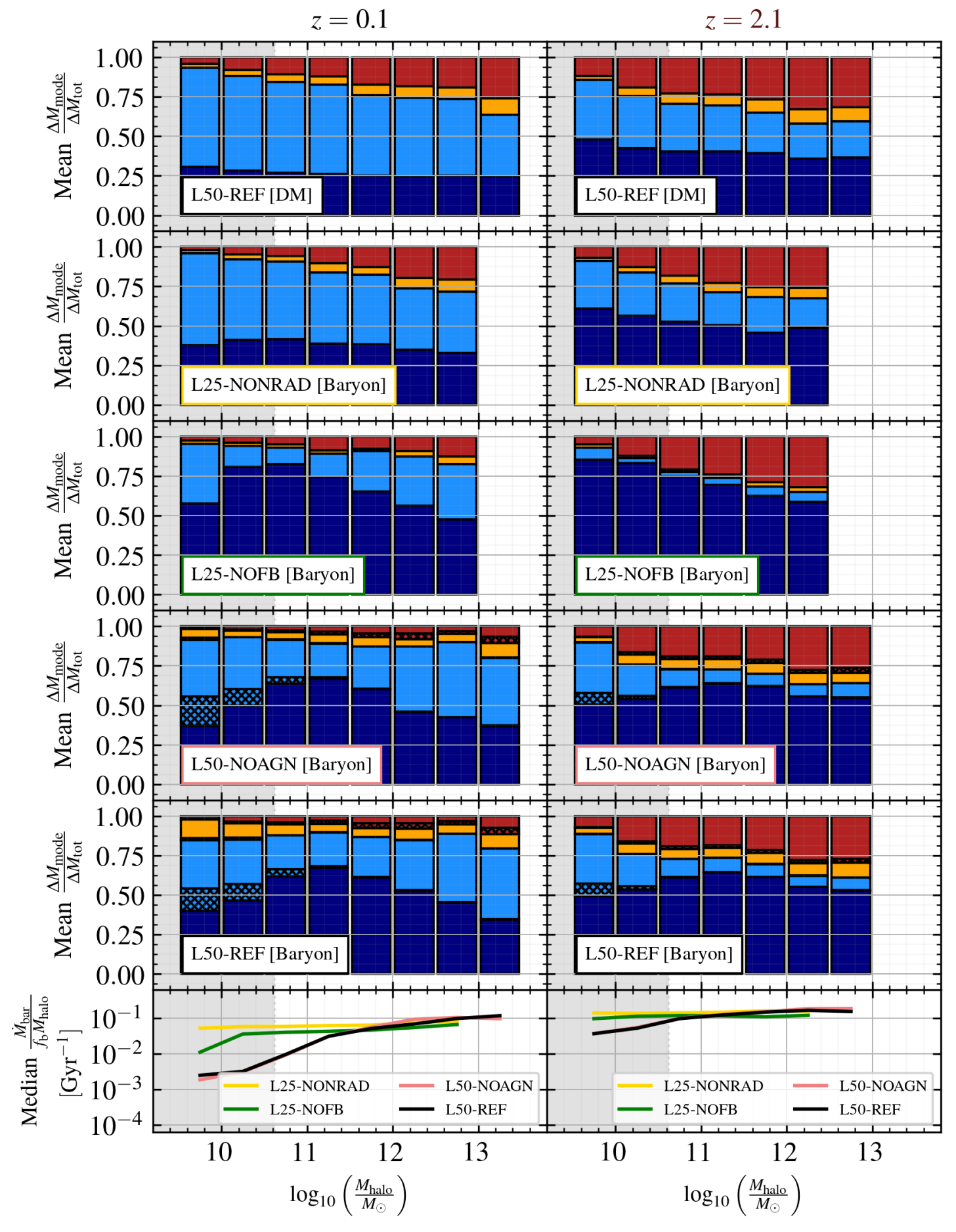

First infall $\square$ Recycled $\square$ Transfer $\square$ Merger Stellar contribution

Figure 10. Channels of baryon and DM accretion as a function of halo mass for $z \approx 0$ (left panels) and $z \approx 2$ (right panels). We use the same definitions of accretion channels as outlined in $\S 2.3$ and Figure 6 , and the same halo mass bins as in Figure 6 . In the first row of panels we present our DM results from L50-REF for comparison (with DM channels not significantly changing between runs). In the second-fifth rows of panels, we present the baryon accretion channels for 4 EAGLE variants, from top to bottom: (i) L25-NONRAD, (ii) L25-NOFB, (iii) L50-NOAGN, and (iv) L50-REF. The bottom row of panels shows the median gas accretion efficiency in each of these bins, to remind the reader of the relative normalisation of raw accretion rates as a function of halo mass for these different runs. We show that the sequential introduction of radiative cooling, stellar and AGN feedback influences inflow channels considerably relative to DM-only physics. 
fall baryon accretion that we previously saw with reference physics in Figure 6, where recycling would otherwise dominate. This drastic change tells us that the enhanced recycling seen in L25-NONRAD is a result of non-radiative physics. While we have not directly investigated particle trajectories, in the non-radiative runs we believe that more thermal pressure around the FOF boundary means that particles may enter the halo and promptly "bounce" back outside the boundary. When these particles are eventually re-accreted, this second accretion will be considered recycled. With radiative cooling, the pressure support at the halo boundary is reduced, meaning that first infall particles are more likely to stay in the halo, and not be re-accreted at a later time. At at $z \approx 2$ in L25-NOFB, baryons are slightly more likely to be accreted via mergers (i.e., less likely to be smoothly accreted) compared to DM in higher mass halos $\left(M_{\text {halo }} \gtrsim 10^{11.5} M_{\odot}\right)$. This is naturally expected in a run implementing radiative cooling, as these processes allow baryons to accumulate at the centres of haloes in a run-away fashion - also known as overcooling (White \& Rees 1978) - increasing the amount of baryon mass contributed by mergers.

In the 4th row of panels, we introduce stellar feedback in a larger box with the L50-NOAGN run. An interesting result here is that the baryon growth from mergers drops by a factor of $\approx 2$ across halo mass compared to L25-NOFB at $z \approx 0$, producing the final small merger contribution we saw in Figure 6. As discussed in $\S 3.2 .2$ and 4.1, this is a consequence of stellar feedback suppressing gas inflow onto low mass haloes (see bottom panels), causing them to be baryonpoor relative to the universal $f_{\mathrm{b}}$. Subsequent mergers involving these baryon-poor haloes provide predominantly DM mass, and minimal baryonic matter. The recycled component in L50-NOAGN remains suppressed compared to DM, but is slightly increased compared to baryons in L25-NOFB - a direct result of the ejection and re-accretion of baryons driven by stellar feedback. We observe a small increase in the transfer channel of accretion in L50-NOAGN compared to L25-NOFB, which we attribute to particles being ejected by particularly strong stellar feedback events and later reaccreted to un-related haloes. It appears that high-mass halos, $M_{\text {halo }} \gtrsim 10^{12} M_{\odot}$, are slightly more likely to see enhanced transferred accretion, a likely result of their deeper potential well and ability to attract particles previously ejected from smaller haloes, where stellar feedback-driven mass loading is largest (Mitchell et al. 2020b).

Finally, in the 5th row of panels, we introduce AGN feedback with L50-REF to reach the results we presented in $\S 3.2 .2$. There are minimal changes to observe with reference physics comparing to L50-NOAGN, however, we see a slight change in that baryons are slightly less likely to be recycled and more likely to be transferred relative to L50AGN at $10^{12} M_{\odot} \lesssim M_{\text {halo }} \lesssim 10^{13} M_{\odot}$ for the $z \approx 0$ panel. This is due to AGN feedback boosting the outflow rates in massive haloes by factors of $3-10$ (with the exact value increasing with decreasing redshift; Mitchell et al. 2020b). Because the momentum and energy injection onto outflows by AGN feedback is $\gtrsim 3$ times larger than stellar feedback at $z<1$ in these massive haloes for the EAGLE reference physics (Mitchell et al. 2020b), the ejected gas particles can move further away from the potential well of these massive haloes. A fraction of those particles are likely to escape the potential well and not make its way back to re-accretion.
Without AGN feedback, this does not happen, and effectively all ejected particles from haloes are expected to be re-accreted by the same halo. Additionally, the higher virial temperature associated with massive haloes prevents reincorporation of AGN-ejected gas due to (i) direct thermal support in the intra-cluster medium (ICM), and (ii) less efficient cooling (given halo virial temperatures are above the peak of the radiative cooling curve).

\section{IMPLICATIONS FOR MODELLING GALAXY EVOLUTION}

In this section, we explore the implications of our results in $\S 3$ and $\S 4$ on our understanding of galaxy evolution, and their application to SAMs. We first discuss the dependence of our results on the model used. While we have established that there is significant feedback-induced suppression of halo-scale gas inflow onto low-mass haloes in EAGLE, the scale at which inflows interact with outflows and the dynamics of this interface are not well understood.

Mitchell et al. (2020b) compare outflow rates and mass loading on various scales in EAGLE with other hydrodynamical simulations (e.g. from Illustris-TNG - Nelson et al. 2019; Horizon-AGN - Beckmann et al. 2017). They find that although trends across halo mass are qualitatively similar, the biggest uncertainty comes from the scale over which outflows are measured. At $M_{\star} \approx 10^{9} M_{\odot}$, outflow rates in Illustris-TNG appear to be stifled between 10 and $50 \mathrm{kpc}$, while outflow flux remains the same in EAGLE at $50 \mathrm{kpc}$ compared to $10 \mathrm{kpc}$. In general, Illustris-TNG seems to prefer a galactic-fountain picture with strong recycling over smaller scales. EAGLE, on the other hand, produces outflows that remove comparatively less material from galaxies, but that become significantly entrained as they expand in the haloes (resulting in the ejection of material to much larger radii compared to Illustris-TNG, Mitchell et al. 2020b). As such, it is important for us to note that simulation-based halo-scale inflow measurements, and the impact of sub-grid physics, are almost certainly strongly model-dependent (as is explored further in Mitchell et al. 2020a). Similarly, variations in the baryon inflow rate onto haloes are also model-dependent. This highlights the need for observational constraints on the physical properties of accreting gas (temperature, density and metallicity), and in an upcoming paper (Wright et al. in prep), we show how these physical properties are sensitive to both the sub-grid physics implemented, and the channel through which the gas is accreting by. These differences may be a promising way of using observations to indirectly constrain the effect of outflows and inflows at the scale of haloes and beyond.

Moving onto the implications of our results for SAMs, we first remind the reader that most SAMs use merger trees produced from DM-only simulations as a base to form and evolve galaxies, with haloes growing their baryonic mass based on the net change in DM mass (modulo a factor of $f_{\mathrm{b}}$ ). The baryon budget within the halo is then managed analytically, and evolved with the specific models implemented within the SAM. Our work highlights a seldom-addressed issue comparing mass growth between SAMs and hydrodynamical simulations, with the significance of DM recycling indicating that gross DM accretion rates do not necessarily 
reflect net DM growth within a halo. One could argue that it is more appropriate to model baryon growth based only on first infall DM accretion rates (which would require haloby-halo accretion calculations compared to a simple $\Delta M$ calculation). We defer a full discussion of this topic to a future paper.

While halo-scale inflow has been shown to be less sensitive to baryonic feedback compared to galaxy-scale inflow (e.g. van de Voort et al. 2011, Nelson et al. 2015), we argue that the suppression of halo gas inflow with the inclusion of stellar feedback (by up to $\approx 1$ dex for lower mass haloes, $<10^{11} \mathrm{M}_{\odot}$, see Figure 9) is significant enough to have implications on the treatment of baryons in SAMs. Most SAMs implement some form of stellar and AGN feedback by ejecting gas from galaxies into the CGM, or from the halo entirely. We have shown here, however, that feedback plays a dual role in not only ejecting baryons from galaxies, but also preventing subsequent inflow onto their host halo. We would expect this effect to be significant in haloes in the mass range $M_{\text {halo }} \lesssim 10^{12} M_{\odot}$ (corresponding to $M_{\star}$, cen $\left.\lesssim 10^{10} M_{\odot}\right)$, where baryon inflow is $\lesssim 50 \%$ of that expected from DM (at $z \lesssim 1$ ). Our resolution with L50-REF limits us to only making this conclusion for haloes of mass $M_{\text {halo }} \gtrsim 10^{10.5} M_{\odot}$ (corresponding to $M_{\star}$, cen $\approx 10^{7.5} M_{\odot}$ ), however we can confidently see this suppression continuing below this mass (to $M_{\text {halo }} \approx 10^{10} M_{\odot}$, where $M_{\star}$, cen $\left.\approx 10^{7} M_{\odot}\right)$ in the L25N752RECAL run; see Appendix Figure B1.

In the aforementioned mass range $\left(M_{\text {halo }} \lesssim 10^{12} M_{\odot}\right)$ that we have established there to be baryon depleted inflow, haloes in current SAMs are subject to over-predicted gas inflow rates by up to 1 dex. Mitchell et al. (2018) showed that halo baryon fractions in EAGLE are far lower than those found in GALFORM for haloes with mass $\lesssim 10^{12.5} M_{\odot}$. Our findings contextualise this result, showing that these overly baryon rich haloes in SAMs could be plausibly explained by over-predicted baryon growth. This puts pressure on feedback mechanisms in SAMs, particularly stellar feedback, to remove the excess baryons in order to reproduce observational standards. While SAMs have had success in reproducing a number of calibrations from observations (e.g. the stellar mass function, see Henriques et al. 2015; Lacey et al. 2016; Lagos et al. 2018b), it has been shown that they do so for very different reasons compared to hydrodynamical simulations (Mitchell et al. 2018).

Lu et al. (2017) show, with a semi-analytic approach (outlined in Lu et al. 2016), that an exclusively "ejective"mode feedback implementation cannot simultaneously recover the mass-metallicity relation (MMR) and stellar mass function found in low mass Milky Way dwarfs. They argue that with the strong stellar-driven outflows required to suppress star formation also comes the ejection of metals, eventually producing an artificially steep MMR that predicts lower metallicity dwarfs than observed. On the other hand, hydrodynamical simulations, which naturally include the "preventative" effect of feedback and suppression of gas inflow (not just the "ejective" effect), are able to reproduce the shape of the observed MMR fairly robustly where innerhaloes retain adequate metal content (e.g. EAGLE - Schaye et al. 2015; Illustris - Torrey et al. 2014). Agertz et al. (2020) highlight the ability of the MMR in assessing the accuracy of models, showing that changes to stellar feedback significantly influence the MMR without necessarily influencing other commonly used scaling relations (e.g. involving half-mass radii and stellar velocity dispersions). Our results further support the notion that "preventative"-mode feedback (in particular, stellar feedback-induced suppression of baryon inflow), in addition to traditional "ejective"-mode feedback, is necessary to accurately model galaxy formation and evolution in SAMs: not just at the galaxy-scale, but also at the halo-scale.

Lastly, as briefly discussed in $\S 3.3$, we show that satellite subhaloes are certainly still capable of accreting cosmological gas. Most SAMs will assume that satellite galaxies are cut off from cosmological accretion upon infall to a larger host halo: an assumption which we plan to further investigate in a future paper, with an analysis of the conditions under which satellite subhaloes are able to net accrete baryonic matter.

The above discussion points to the fact that feedback has two-fold profound influence on halo-scale baryonic mass assembly - in (i) removing baryonic matter from galaxies and haloes, and then (ii) preventing further baryonic inflow. This level of complexity (particularly the preventative aspect) is not captured by SAMs, and important revisions are therefore required for these tools to accurately model the effects that we analysed in this paper.

\section{SUMMARY}

In this paper we have presented measurements of halo-scale baryon and DM inflow rates in the EAGLE suite of simulations. Our FOF-based algorithm, CHUMM, recovers gas inflow efficiencies $\left(\dot{M}_{\text {gas }} / f_{\mathrm{b}} M_{\text {halo }}\right)$ that are in broad quantitative agreement presented in previous literature using hydrodynamical galaxy formation simulations (e.g. van de Voort et al. 2011, Correa et al. 2018) to within $\approx 0.2$ dex at both $z \approx 0$ and $z \approx 2$ (see Figure 2 ). We consider accretion in 4 different channels: (i) first infall (accreting particles which have never been identified as part of a halo in the past), (ii) recycled-mode (particles which have been processed in a progenitor halo beforehand, but were most recently accreted from the field) (iii) transfer (particles which have been processed in a non-progenitor/unrelated halo beforehand, but were most recently accreted from the field), and (iv) mergermode (particles which were accreted to a halo which, at the previous snapshot, were part of another halo). Our main findings using the fiducial physics EAGLE run L50-REF were presented in $\S 3$, and are summarised below:

- Baryon accretion is suppressed relative to DM in low mass field haloes $\left(M_{\text {halo }} \lesssim 10^{12} M_{\odot}\right.$, corresponding to $M_{\star}$, cen $\left.\lesssim 10^{10} M_{\odot}\right)$ by up to 1 dex (see Figure 3 ). This effect is particularly prominent towards late times, where the halo-to-halo variance in $f_{\mathrm{b} \text {, inflow }}=\dot{M}_{\mathrm{bar}} /\left(\dot{M}_{\mathrm{bar}}+\dot{M}_{\mathrm{DM}}\right)$ is also largest. In the same mass range, the baryon fractions of halo-scale inflow are very well-correlated with halo-wide baryon fractions (most notably in the CGM), suggesting a causal effect (see Figures 4 and 5).

- Regarding accretion channels:

(i) The merger-mode growth channel is of lesser importance for baryons compared to DM in more massive haloes 
$\left(M_{\text {halo }} \gtrsim 10^{12} M_{\odot}\right)$, particularly at late times. This is directly attributable to the baryon depletion of the low mass haloes that contribute mass in mergers (see Figure 6). Baryon depletion of low mass haloes could either be due to the aforementioned baryon poor inflow, or stripping \& pre-processing in the case of satellites, see Bahé \& McCarthy 2015; Bahé et al. 2019), which are only capable of contributing significant mass in the form of DM during merger events.

(ii) Compared to baryonic matter, smoothly accreted DM is significantly more likely (by a factor of $\approx 2$ ) to have been recycled in a halo than to be on first infall (with the exception of higher mass haloes, $M_{\text {halo }} \gtrsim 10^{12.5} M_{\odot}$, at $z \approx 0$ ). This is a reflection of the ubiquitous suppression of baryon inflow onto low mass haloes over cosmic time: any given baryon particle is less likely to have been accreted at some point in the simulation compared to a DM particle.

- Inflow rates to satellite subhaloes are influenced by their stellar-to-halo mass ratio $\left(M_{\star} / M_{\text {halo }}\right)$ and their normalised halo-centric distance $\left(R_{\mathrm{sat}}\right.$, halo $\left./ R_{200 \text {, halo }}\right)$ with more massive satellites and satellites at larger radii relative to their host being more likely to see higher gas accretion rates (Figure $7)$.

In $\S 4$, we go on to investigate the influence of sub-grid physics on the results that we summarise above, by making use of several additional simulation runs (outlined in Tables 1 and 2). We find that:

- In EAGLE, the significant suppression (by up to $\approx 1$ dex) of $f_{\mathrm{b}}$, inflow in low mass haloes $\left(M_{\text {halo }} \lesssim 10^{12} M_{\odot}\right)$ compared to the universal $f_{\mathrm{b}}$ is a direct result of the introduction of stellar feedback (see Figures 8, 9). In addition, the interplay between stellar and AGN feedback in EAGLE has a profound influence on gas inflow. Weakening stellar feedback can lead to runaway SMBH accretion and subsequent AGN outflow, which can heavily suppress gas accretion rates (for an in-depth explanation of the interaction between stellar and AGN feedback in EAGLE, see Bower et al. 2017). For a fixed stellar feedback implementation, we see that altering AGN feedback injection temperature ("explosivity") can modulate gas inflow rates in high mass haloes $\left(M_{\text {halo }} \gtrsim 10^{12.5} M_{\odot}\right.$ by $\left.\approx 25-50 \%\right)$ - see Figures 8 and 9 .

- Gas inflow channels are strongly influenced by sub-grid physics (Figure 10). Gas and DM are accreted onto haloes with similar contributions from both smooth/merger and recycled/first infall channels in the runs with no radiative cooling, star formation and feedback. Introducing radiative cooling leads to a decrease in the gas recycling fractions, which we attribute to gas cooling removing the thermal pressure at the virial radius that naturally builds up in the non-radiative runs. Adding stellar feedback with the L50NOAGN run increases the importance of the baryon recycling and transfer accretion channels at all halo masses as a result of supernovae-driven outflows, which are eventually re-accreted onto the halo - see Figure 10. Introducing AGN feedback with L50-REF has little influence on the importance of the different accretion channels analysed here compared to the L50-NOAGN run, however marginally reduces the recycling-mode and increases inter-halo transfermode of baryon inflow for high mass haloes, $M_{\text {halo }} \gtrsim 10^{12} M_{\odot}$, which we attribute to AGN outflows being sufficiently strong to entirely eject gas particles from haloes, without eventual reincorporation.

In $\S 5$, we discuss the model-dependence of our results, and the implications that our findings have on semianalytic approaches with supporting literature. We argue that preventative-mode feedback (i.e., suppressing gas inflow), in addition to traditional ejective-mode feedback (i.e., energetic gas outflows) are both important manifestations of stellar feedback in EAGLE. This dual effect (even at the halo-scale) seems to be required to accurately model the behaviour of low mass haloes (with a particularly useful calibration of stellar feedback being the mass-metallicity relation - see Lu et al. 2017; Agertz et al. 2020).

We point the reader towards the work of Mitchell et al. (2020a) for an in-depth analysis of the physics of accretion in EAGLE towards the CGM- and galaxy-scale, together with the interplay between outflows, inflows and recycling. In a future paper, we will look to further investigate gas accretion onto subhaloes in hydrodynamical simulations: specifically, the conditions under which subhaloes are able to net grow in baryonic mass. This is a topic of of particular interest for SAMs, as most will assume that satellite galaxies are abruptly cut off from cosmological accretion upon infall to a host. Additionally, in another future paper, we plan to examine the properties of accreting baryons at the halo-scale: specifically the temperature, density, and metallicity of inflow particles around the virial radius. We will investigate the sensitivity of these properties to sub-grid physics implementations, which could have observational applications in being able to constrain the effect of outflows and inflows at the halo-scale.

\section{ACKNOWLEDGEMENTS}

RW is funded by a Postgraduate Research Scholarship from the University of Western Australia (UWA). CL is funded by the ARC Centre of Excellence for All Sky Astrophysics in 3 Dimensions (ASTRO 3D), through project number CE170100013. CL also thanks the MERAC Foundation for a Postdoctoral Research Award. CP acknowledges the support ASTRO 3D. PM acknowledges a UWA Research Collaboration Award 2018 for funding his visit to UWA. This work made use of the supercomputer OzSTAR, which is managed through the Centre for Astrophysics \& Supercomputing, and maintained by Swinburne ITS. Their supercomputing program receives continued financial support for operations from Astronomy Australia Limited and the Australian Commonwealth Government through the National Collaborative Research Infrastructure Strategy (NCRIS). The EAGLE simulations were performed using the DiRAC-2 facility at Durham, managed by the ICC, and the PRACE facility Curie based in France at TGCC, CEA, Bruyeres-le-Chatel.

\section{REFERENCES}

Agertz O., et al., 2020, MNRAS, 491, 1656

Anglés-Alcázar D., Faucher-Giguère C.-A., Kereš D., Hopkins P. F., Quataert E., Murray N., 2017, MNRAS, 470, 4698

Bahé Y. M., McCarthy I. G., 2015, MNRAS, 447, 969

Bahé Y. M., et al., 2019, MNRAS, 485, 2287 
Beckmann R. S., et al., 2017, MNRAS, 472, 949

Behroozi P., Wechsler R. H., Hearin A. P., Conroy C., 2019, MNRAS, 488, 3143

Benson A. J., 2010, Phys. Rep., 495, 33

Binney J., 1977, ApJ, 215, 483

Bouché N., et al., 2010, ApJ, 718, 1001

Bouché N., Murphy M. T., Kacprzak G. G., Péroux C., Contini T., Martin C. L., Dessauges-Zavadsky M., 2013, Science, 341, 50

Bouché N., et al., 2016, ApJ, 820, 121

Bower R. G., Schaye J., Frenk C. S., Theuns T., Schaller M., Crain R. A., McAlpine S., 2017, MNRAS, 465, 32

Chabrier G., 2003, PASP, 115, 763

Cole S., Lacey C. G., Baugh C. M., Frenk C. S., 2000, MNRAS, 319,168

Collacchioni F., Lagos C. D. P., Mitchell P. D., Schaye J., Wisnioski E., Cora S. A., Correa C. A., 2019, arXiv e-prints, p. arXiv:1910.05377

Correa C. A., Wyithe J. S. B., Schaye J., Duffy A. R., 2015a, MNRAS, 450, 1514

Correa C. A., Wyithe J. S. B., Schaye J., Duffy A. R., 2015b, MNRAS, 450, 1521

Correa C. A., Wyithe J. S. B., Schaye J., Duffy A. R., 2015c, MNRAS, 452, 1217

Correa C. A., Schaye J., van de Voort F., Duffy A. R., Wyithe J. S. B., 2018, MNRAS, 478, 255

Crain R. A., Eke V. R., Frenk C. S., Jenkins A., McCarthy I. G., Navarro J. F., Pearce F. R., 2007, MNRAS, 377, 41

Crain R. A., et al., 2015, MNRAS, 450, 1937

Dalla Vecchia C., Schaye J., 2012, MNRAS, 426, 140

Davé R., Finlator K., Oppenheimer B. D., 2012, MNRAS, 421, 98

Davies J. J., Crain R. A., McCarthy I. G., Oppenheimer B. D., Schaye J., Schaller M., McAlpine S., 2019, MNRAS, 485, 3783

Davis M., Efstathiou G., Frenk C. S., White S. D. M., 1985, ApJ, 292,371

Dekel A., Birnboim Y., 2006, MNRAS, 368, 2

Dekel A., et al., 2009, Nature, 457, 451

Dolag K., Borgani S., Murante G., Springel V., 2009, MNRAS, 399, 497

Elahi P. J., Thacker R. J., Widrow L. M., 2011, MNRAS, 418, 320

Elahi P. J., Cañas R., Poulton R. J. J., Tobar R. J., Willis J. S., Lagos C. d. P., Power C., Robotham A. S. G., 2019a, Publ. Astron. Soc. Australia, 36, e021

Elahi P. J., Poulton R. J. J., Tobar R. J., Cañas R., Lagos C. d. P., Power C., Robotham A. S. G., 2019b, Publ. Astron. Soc. Australia, 36, e028

Emonts B. H. C., et al., 2015, A\&A, 584, A99

Faucher-Giguère C.-A., 2017, in Fox A., Davé R., eds, Astrophysics and Space Science Library Vol. 430, Gas Accretion onto Galaxies. p. 271 (arXiv:1612.00448), doi:10.1007/9783-319-52512-9_12

Genel S., Bouché N., Naab T., Sternberg A., Genzel R., 2010, ApJ, 719, 229

Haardt F., Madau P., 2001, in Neumann D. M., Tran J. T. V., eds, Clusters of Galaxies and the High Redshift Universe Observed in X-rays. p. 64 (arXiv:astro-ph/0106018)

Henriques B. M. B., White S. D. M., Thomas P. A., Angulo R., Guo Q., Lemson G., Springel V., Overzier R., 2015, MNRAS, 451,2663

Ho S. H., Martin C. L., Kacprzak G. G., Churchill C. W., 2017, ApJ, 835, 267

Jenkins A., 2013, MNRAS, 434, 2094

Kacprzak G. G., Pointon S. K., Nielsen N. M., Churchill C. W., Muzahid S., Charlton J. C., 2019, ApJ, 886, 91

Katz N., Keres D., Dave R., Weinberg D. H., 2003, in Rosenberg J. L., Putman M. E., eds, Astrophysics and Space Science
Library Vol. 281, The IGM/Galaxy Connection. The Distribution of Baryons at $\mathrm{z}=0$. p. 185 (arXiv:astro-ph/0209279), doi:10.1007/978-94-010-0115-1_34

Kennicutt Jr. R. C., 1998, ApJ, 498, 541

Kereš D., Katz N., Weinberg D. H., Davé R., 2005, MNRAS, 363, 2

Kereš D., Katz N., Fardal M., Davé R., Weinberg D. H., 2009, MNRAS, 395, 160

Lacey C. G., et al., 2016, MNRAS, 462, 3854

Lagos C. d. P., Schaye J., Bahé Y., Van de Sande J., Kay S. T., Barnes D., Davis T. A., Dalla Vecchia C., 2018a, MNRAS, 476,4327

Lagos C. d. P., Tobar R. J., Robotham A. S. G., Obreschkow D., Mitchell P. D., Power C., Elahi P. J., 2018b, MNRAS, 481, 3573

Lehner N., Howk J. C., 2011, Science, 334, 955

Lilly S. J., Carollo C. M., Pipino A., Renzini A., Peng Y., 2013, ApJ, 772, 119

Lockman F. J., Benjamin R. A., Heroux A. J., Langston G. I., 2008, ApJ, 679, L21

Lu Y., Benson A., Mao Y.-Y., Tonnesen S., Peter A. H. G., Wetzel A. R., Boylan-Kolchin M., Wechsler R. H., 2016, ApJ, 830, 59

Lu Y., Benson A., Wetzel A., Mao Y.-Y., Tonnesen S., Peter A. H. G., Boylan-Kolchin M., Wechsler R. H., 2017, ApJ, 846, 66

Martin C. L., Ho S. H., Kacprzak G. G., Churchill C. W., 2019, ApJ, 878, 84

McAlpine S., et al., 2016, Astronomy and Computing, 15, 72

Mitchell P. D., et al., 2018, MNRAS, 474, 492

Mitchell P. D., Schaye J., Bower R. G., 2020a, arXiv e-prints, p. arXiv:2005.10262

Mitchell P. D., Schaye J., Bower R. G., Crain R. A., 2020b, MNRAS, 494, 3971

Moster B. P., Naab T., White S. D. M., 2018, MNRAS, 477, 1822

Neistein E., van den Bosch F. C., Dekel A., 2006, MNRAS, 372, 933

Nelson D., Genel S., Vogelsberger M., Springel V., Sijacki D., Torrey P., Hernquist L., 2015, MNRAS, 448, 59

Nelson D., et al., 2019, MNRAS, 490, 3234

Nielsen N. M., Kacprzak G. G., Pointon S. K., Murphy M. T., Churchill C. W., Davé R., 2020, arXiv e-prints, p. arXiv:2002.08516

Ocvirk P., Pichon C., Teyssier R., 2008, MNRAS, 390, 1326

Oppenheimer B. D., et al., 2020, MNRAS, 491, 2939

Peng Y.-j., Maiolino R., 2014, MNRAS, 443, 3643

Pereira-Santaella M., et al., 2016, A\&A, 594, A81

Planck Collaboration et al., 2014, A\&A, 571, A16

Planck Collaboration et al., 2018, arXiv e-prints, p. arXiv:1807.06209

Pointon S. K., Kacprzak G. G., Nielsen N. M., Muzahid S., Murphy M. T., Churchill C. W., Charlton J. C., 2019, ApJ, 883, 78

Rahmani H., et al., 2018, MNRAS, 474, 254

Rees M. J., Ostriker J. P., 1977, MNRAS, 179, 541

Rubin K. H. R., 2017, Gas Accretion Traced in Absorption in Galaxy Spectroscopy. Springer International Publishing, p. 95, doi:10.1007/978-3-319-52512-9_5

Rubin K. H. R., Prochaska J. X., Koo D. C., Phillips A. C., 2012, ApJ, 747, L26

Schaller M., Dalla Vecchia C., Schaye J., Bower R. G., Theuns T., Crain R. A., Furlong M., McCarthy I. G., 2015, MNRAS, 454, 2277

Schaye J., Dalla Vecchia C., 2008, MNRAS, 383, 1210

Schaye J., et al., 2015, MNRAS, 446, 521

Somerville R. S., Davé R., 2015, Annual Review of Astronomy and Astrophysics, 53, 51

Springel V., 2005, MNRAS, 364, 1105 
Springel V., Yoshida N., White S. D. M., 2001, New Astron., 6, 79

Springel V., Di Matteo T., Hernquist L., 2005, ApJ, 620, L79

Torrey P., Vogelsberger M., Genel S., Sijacki D., Springel V., Hernquist L., 2014, MNRAS, 438, 1985

White S. D. M., Rees M. J., 1978, MNRAS, 183, 341

Wiersma R. P. C., Schaye J., Theuns T., Dalla Vecchia C., Tornatore L., 2009, MNRAS, 399, 574

Wright R. J., Lagos C. d. P., Davies L. J. M., Power C., Trayford J. W., Wong O. I., 2019, MNRAS, 487, 3740

Zabl J., et al., 2019, MNRAS, 485, 1961

van de Voort F., Schaye J., Booth C. M., Haas M. R., Dalla Vecchia C., 2011, MNRAS, 414, 2458

van de Voort F., Bahé Y. M., Bower R. G., Correa C. A., Crain R. A., Schaye J., Theuns T., 2017, MNRAS, 466, 3460

\section{APPENDIX A: FIELD HALO MASS DISTRIBUTIONS}

With the aim of providing the reader a measure of the sample sizes we use to draw conclusions from, we show in Figure A1 the number of field haloes as a function of halo mass for various runs used throughout the paper. The bin size we use is identical to that used throughout the rest of the paper (unless otherwise stated).

\section{APPENDIX B: RESOLUTION CONVERGENCE}

Schaye et al. (2015) introduced the concept of "strong" and "weak" convergence tests. Strong convergence refers to the case where a simulation is re-run at higher resolution, with both better mass and spatial resolution, adopting exactly the same sub-grid physics models and parameters. Weak convergence refers to the case when a simulation is re-run at higher resolution but the sub-grid parameters are recalibrated to recover, as best as possible, the level of agreement with the adopted calibration diagnostic. In the case of EAGLE, the $z=0.1$ galaxy stellar mass function and stellar size-mass relation of galaxies. With this purpose, two higher-resolution versions of EAGLE were introduced by Schaye et al. (2015). Both in a box of $(25 \mathrm{cMpc})^{3}$ and with $2 \times 752^{3}$ particles. These simulations have better mass and spatial resolution than the intermediate-resolution of the L50-REF simulation by factors of 8 and 2, respectively. The EAGLE L25N752REF adopts identical parameters to the standard resolution reference runs, whereas L25N752-RECAL has 4 parameters that were recalibrated to reproduce $z=0$ observables above. Hence, a comparison between L25N376-REF and L25N752REF represents a strong convergence test, while a comparison with L25N752-RECAL is a weak convergence test. Here, we compare with both high resolution runs to examine the convergence of our results.

Figure B1 shows accretion rates (left) and inflow baryon fractions (right) as a function of halo mass for the L25N752RECAL, L25N752-REF and L25N376-REF (we also include L50-REF in black). Focusing on raw accretion rates (left panels), DM accretion rates agree very well between different resolutions, and show very little spread $(\lesssim 0.5$ dex across all halo masses), while gas accretion rates are systematically enhanced in L25N752-REF and L25N752-RECAL compared to $\mathrm{L} 50-\mathrm{REF}$ by $\approx 0.1-0.2 \mathrm{dex}$. The natural spread in gas accretion rates increases towards lower halo masses for the 3 runs shown, however the magnitude of the spread at a given halo mass is slightly less in the higher resolution runs. In the right panels we illustrate $f_{\mathrm{b}}$, inflow using our primary FOF method (solid lines), and also using the $R_{200}$ spherical overdensity method (densely dotted lines, only for L50-REF and L25N752-RECAL). Similar to raw accretion rates, we see that baryon accretion rates relative to DM are systematically enhanced in L25N752-REF and L25N752-RECAL compared to L50-REF by $\approx 0.1-0.2 \mathrm{dex}$ (the offset fairly steady over halo mass). We see the same enhancement of gas accretion using our $R_{200}$ method, indicating that this result is not a consequence of methodology, and is rather a physically meaningful reflection of the different accretion rates between the simulations. Although enhancement of gas accretion is seen with higher resolution, the decrease in gas accretion relative to DM remains significant nonetheless. We therefore remain confident in our conclusions regarding $f_{\mathrm{b}}$, inflow using the standard resolution L50-REF run. Enhanced gas accretion rates in the higher resolution runs offers an explanation for differences between these two simulations previously reported. Figure 11 in Schaye et al. (2015) and Figure B1 in Collacchioni et al. (2019) show enhanced specific star formation rates (SSFRs) and accretion rates onto galaxies that leads to star formation, respectively, in the L25N752RECAL run relative to the $100 \mathrm{Mpc}$ reference box for galaxies in the stellar mass range $M_{\star} \lesssim 10^{10} M_{\odot}$. These enhancements are likely the consequence of the enhanced baryon inflow rate at the halo level, which increases the abundance of gas available for eventual star formation. Based on the findings in Figure B1, we find convincing strong/weak convergence between L50-REF and L25N752-REF/L25N752RECAL when measuring DM accretion rates, and slight tension when comparing gas accretion rates (the tension being quantitatively consistent when using recalibrated parameters compared to reference parameters).

\section{APPENDIX C: TEMPORAL CONVERGENCE}

As explained in $\S 2.3$, our accretion rates are calculated over a finite time interval, and our accretion rates are therefore sensitive to the choice of $\Delta t$. In the case of EAGLE, we elect to use adjacent snapshots to constitute the time interval for accretion (corresponding to a $\Delta t$ value closest to $\Delta t \approx 1$ Gyr at $z \approx 0$, or, more generally, $\left.\Delta t \approx 0.5-1 \times t_{\text {dyn }}(z)\right)$. We would consider our results to be converged if our accretion rates represent an accurate time=integrated measure of matter inflow, with minimal contribution from stochastic particle accretion and outflow events over short timescales $\left(\ll t_{\text {dyn }}(z)\right)$. Figure $\mathrm{C} 1$ shows accretion efficiencies at $z \approx 0$ in the L32-NONRAD run as a function of halo mass, for a number of different $\Delta t$ interval lengths to investigate the sensitivity of our algorithm to the time interval. We find with adiabatic physics that accretion rates converge towards longer intervals, with shorter $\Delta t$ intervals leading to higher instantaneous accretion rates. This is likely a consequence of more stochastic inwards crossings of the FOF boundary over shorter timescales, while longer $\Delta t$ intervals yield a more accurate time integrated accretion rate. Given our temporal convergence test in Figure C1 using L32-NONRAD, we can be confident that our calculations in EAGLE using longer 


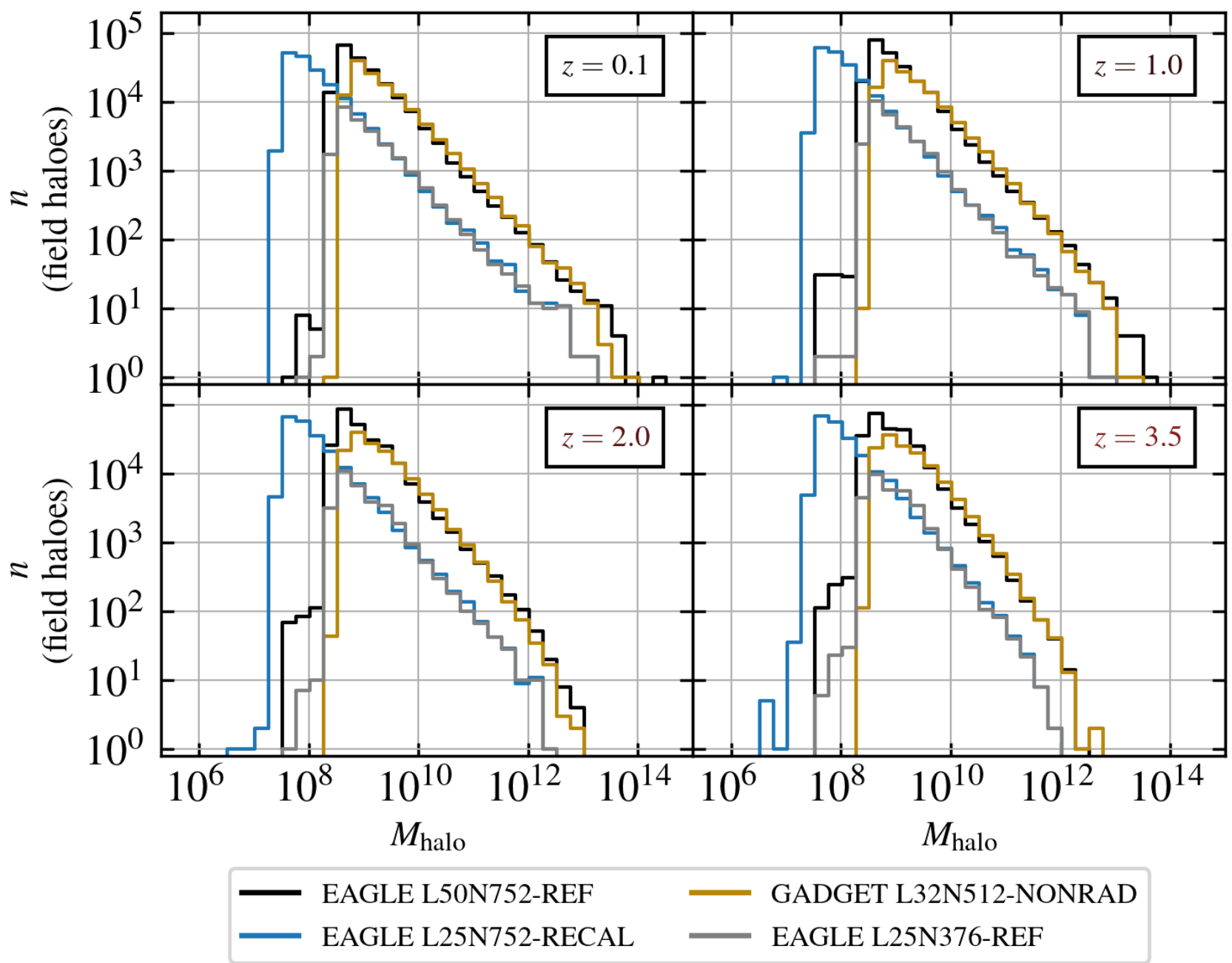

Figure A1. The number of field haloes in a selection of runs as a function of halo mass for 4 redshifts: $z=0.1$ (top left panel), $z=1.0$ (top right panel), $z=2.0$ (bottom left panel), and $z=3.5$ (bottom right panel). In each panel we show data for (i) EAGLE L50N752-REF (black), (ii) GADGET L32N512-NONRAD (khaki), (iii) EAGLE L25N752-RECAL (blue), and (iv) EAGLE L25N376-REF (grey). Any alternative physics runs in Table 1 with 50 (25) Mpc box size show very similar halo mass distributions as L50-REF (L25-REF). We use 36 equally log-spaced bins from $M_{\text {halo }}=10^{6} M_{\odot}$ to $M_{\text {halo }}=10^{15} M_{\odot}$, corresponding to a bin size of 0.25 dex.

$\Delta t$ intervals have converged towards accurate time integrated accretion rates. This is further evidenced in the convergence tests presented in Mitchell et al. (2020a), who show a similar trend using full EAGLE physics. It should also be noted that the behaviour of accretion efficiency with halo mass remains identical comparing different $\Delta t$ values, albeit with different normalisation - meaning that even if the interval we used did not yield accurate time-converged accretion rates, our qualitative results would not be significantly influenced.

\section{APPENDIX D: FOF INFLOW COMPARED TO SO INFLOW}

Figure D1 illustrates the behaviour of our primary FOFbased accretion algorithm (solid black line) compared to an $R_{200}$ spherical mass flux based calculation (coloured lines representing inflow over a collection of fractional $R_{200}$ spheres). We also show accretion efficiencies if we neglect to include accretion rates to satellite subhaloes (dotted line) compared to the standard FOF algorithm (which includes accretion onto satellites). We note that the FOF algorithm neglecting satellite accretion agrees very well with accretion flux to the $R_{200}$ sphere (purple line). Unsurprisingly, including accretion onto satellites increases accretion rates, particularly for higher mass haloes $\left(M_{\text {halo }} \gtrsim 10^{11.5} M_{\odot}\right)$, which are host to more substructure. This increase compared to $R_{200^{-}}$ based inflow is small, of order $\approx 0.1-0.2 \mathrm{dex}$. This comparison gives us confidence in our results, indicating that the influence of the method is both minimal and predictable.

This paper has been typeset from a $\mathrm{T}_{\mathrm{E}} \mathrm{X} / \mathrm{L} \mathrm{LT}_{\mathrm{E}} \mathrm{X}$ file prepared by the author. 

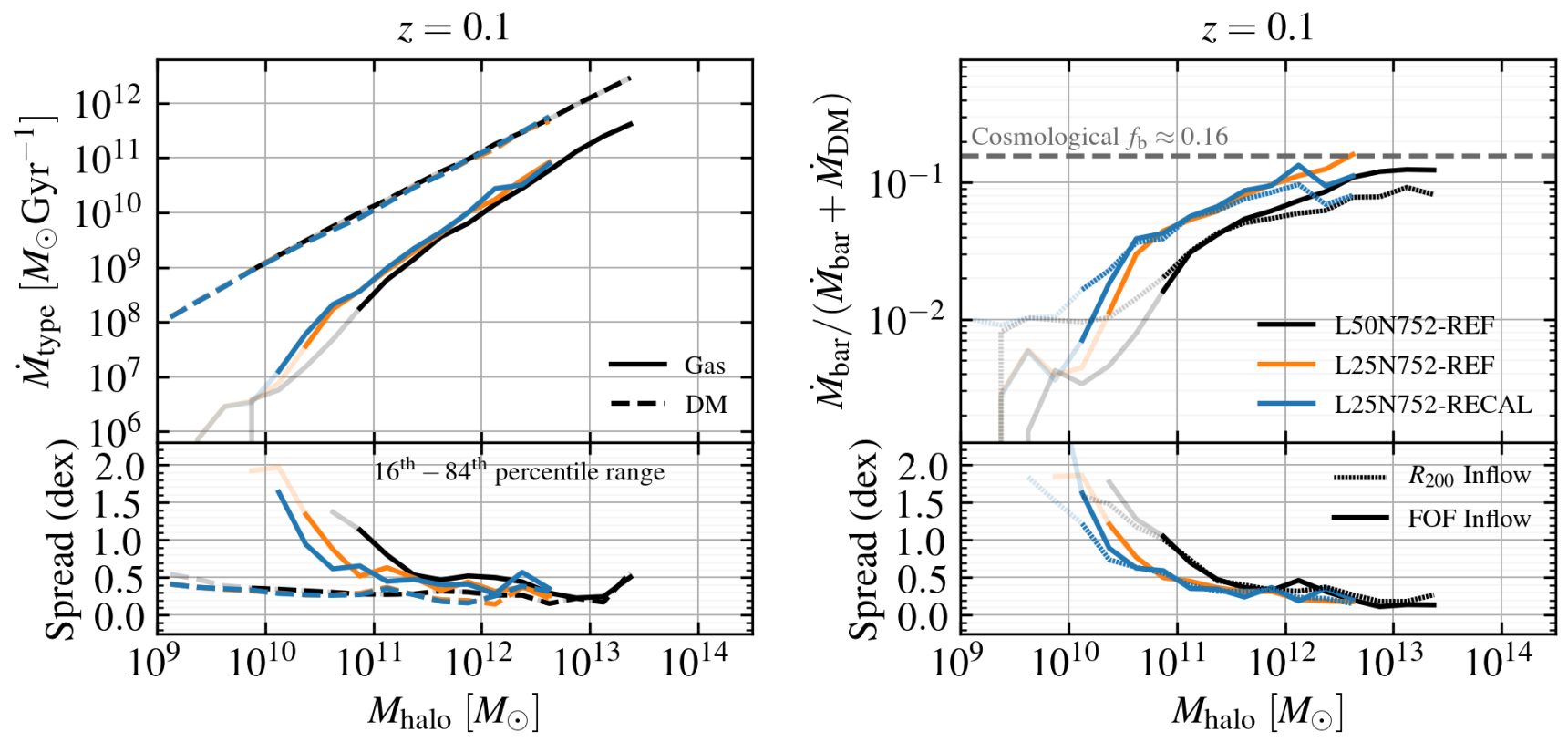

Figure B1. Left panels: Inflow rates (top) and spread (bottom) as a function of halo mass for gas (solid lines) and DM (dashed lines), for the standard resolution EAGLE L50N752-REF box (black) and 2 higher resolution boxes: L25N752-RECAL (blue) and L25N752REF (orange). Right panels: inflow baryon fractions (top) and spread (bottom) as a function of halo mass, for the same runs. We also compare the baryon fractions from an $R_{200}$ inflow algorithm for the L50N752-REF and L25N752-RECAL runs (densely dotted lines). Line transparency has been increased where the average efficiency has been calculated from a bin in which more than $50 \%$ of haloes were subject to an accretion flux of less than 50 particles. We see similar results for DM inflow rates irrespective of resolution, and the same trend for gas accretion onto be suppressed at lower halo masses. We do, however, observe that both higher resolution runs exhibit enhanced gas accretion rates. The spread is marginally reduced for the higher resolution runs at the low mass end. This result is robust using both FOF and $R_{200}$ inflow algorithms.

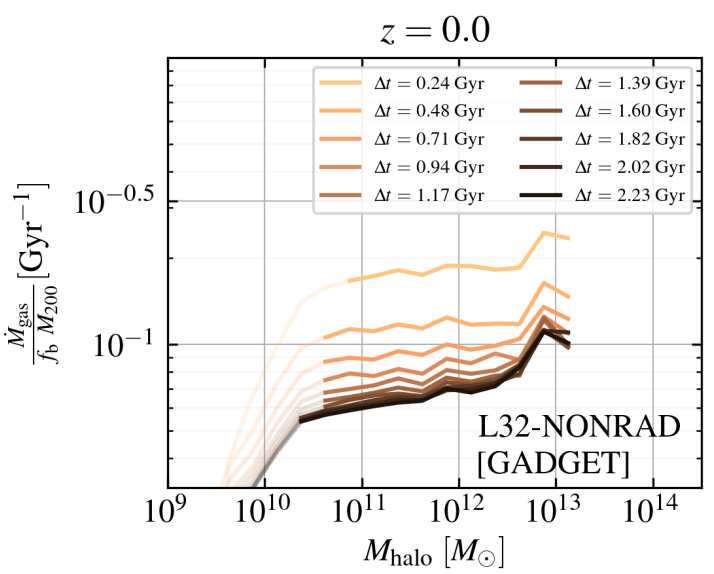

Figure C1. Inflow efficiencies (calculated using a GADGETSPH based non-radiative $32 \mathrm{Mpc}$ box with $512^{3} \mathrm{DM}$ and gas particles) as a function of $M_{200}$ for a collection of $\Delta t$ values (all intervals ending at $z \approx 0$ ). Shorter accretion intervals show increased instantaneous accretion flux, while efficiencies appear to converge towards correct time-integrated inflow flux when we use longer $\Delta t$ values, $\gtrsim 1 \mathrm{Gyr}$, at $z \approx 0$. A similar result is found using full EAGLE physics in Mitchell et al. (2020a). 


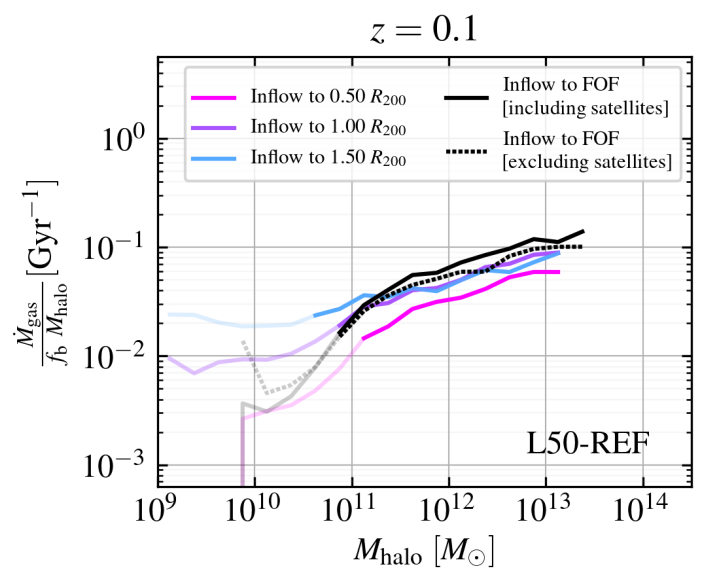

Figure D1. Inflow efficiencies at $z \approx 0$ for the L50-REF box over a collection of $R_{200}$ fractional spheres (coloured lines), compared to (i) FOF inflow including satellites (our primary method, black solid line), and (ii) FOF inflow onto only the central subhalo (i.e. neglecting satellites, black dotted line). Line transparency has been increased where the average efficiency has been calculated from a bin in which more than $50 \%$ of haloes were subject to an accretion flux of less than 50 particles. We observe that our FOF algorithm agrees well with the $\boldsymbol{R}_{200}$ algorithm at halo masses below $10^{11.5} M_{\odot}$, but shows slightly enhanced gas accretion above this mass scale, which we attribute to accretion onto satellite subhaloes. When we only include accretion onto the central subhalo, we also see good agreement with the $R_{200}$ calculation to within 0.1 dex for halo masses above $10^{11.5} M_{\odot}$. 